\title{
RESOURCE ALLOCATION FOR ENERGY HARVESTING ASSISTED D2D COMMUNICATIONS UNDERLAYING OFDMA CELLULAR NETWORKS
}

\author{
by \\ Shuo $\mathrm{Yu}$
}

Bachelor of Engineering, Tianjin University, China, 2015

\author{
A thesis \\ presented to Ryerson University \\ in partial fulfilment of the \\ requirements for the degree of \\ Master of Applied Science \\ in the program of \\ Electrical and Computer Engineering
}

Toronto, Ontario, Canada, 2017

(c) Shuo Yu, 2017 


\section{AUTHORS DECLARATION FOR ELECTRONIC SUBMISSION OF A THESIS}

I hereby declare that I am the sole author of this thesis. This is a true copy of the dissertation, including any required final revisions, as accepted by my examiners.

I authorize Ryerson University to lend this thesis to other institutions or individuals for the purpose of scholarly research.

I further authorize Ryerson University to reproduce this thesis by photocopying or by other means, in total or in part, at the request of other institutions or individuals for the purpose of scholarly research.

I understand that my thesis may be made electronically available to the public. 


\title{
Abstract \\ Resource Allocation for Energy Harvesting Assisted D2D Communications Underlaying OFDMA Cellular Networks
}

\author{
(C) Shuo $\mathrm{Yu}, 2017$ \\ Master of Applied Science \\ Electrical and Computer Engineering \\ Ryerson University
}

D2D communications underlaying cellular networks has become very popular recently. Interference between cellular users and D2D users is one of the tricky issues we need to solve. Another challenging issue is limited battery lives. In this thesis, we address these two issues together by introducing an energy harvesting (EH) assisted D2D model where the whole transmission process is divided into multiple time slots. At the beginning of every time slot, each D2D user will update the remaining energy level to the base station (BS) and the BS will then decide whether the D2D user should harvest energy or transmit data. The objective is to maximize sum throughput for all D2D users. To solve the problem, we first adopt the NOMAD algorithm, then propose a heuristic algorithm as sub-optimal solution. Numerical results show that our proposed algorithm can achieve almost the same sum throughput at a significantly smaller time cost. 


\section{Acknowledgment}

First of all, I would like to thank my supervisors, Alagan Anpalagan and Ling Guan, for their help and support. Every meeting has contributed to the progress of my research and thesis. Their patience and understanding is also what makes this journey more enlightening and rewarding. I also want to thank a fellow researcher in the lab, Dr. Waleed, for his helpful suggestions and all the insightful discussions we had. You will make an amazing professor one day.

Second, I would like to extend my gratitude to the Department of Electrical and Computer Engineering and the School of Graduate Studies of Ryerson University for their financial supports and all the amazing opportunities they have provided for me.

Finally, I would like to thank my parents and friends, without whose help and encouragement I would not be able to achieve my goal and finish my degree. I promise I will continue chasing my dreams and make you proud. 


\section{Contents}

$\begin{array}{ll}\text { Abstract } & \text { iii }\end{array}$

Acknowledgment $\quad$ iv

List of Tables $\quad$ vii

List of Figures viii

List of Abbreviations $\quad$ ix

1 Introduction 1

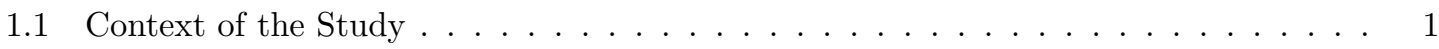

1.2 Objective and Contributions of the Thesis . . . . . . . . . . . . . . 1

1.3 Thesis Organization . . . . . . . . . . . . . . . . . . . . . . . 2

2 Introduction of D2D Communications and Resource Allocation 4

2.1 D2D Communications: Definition, Application and System Architecture . . . . . . . 5

2.2 Categorisation and Comparison of D2D Communications Systems $\ldots \ldots \ldots$

2.3 Resource Allocation Elements in Underlay D2D Communications Systems . . . . . . 11

2.4 Summary . . . . . . . . . . . . . . . . . . . . . . . . . . 13

3 Survey of Resource Allocation Schemes in Underlay D2D communications 14

3.1 Objectives and Constraints of D2D Resource Allocation . . . . . . . . . . . . 15

3.2 Problem Types and Solutions of D2D Resource Allocation . . . . . . . . . . . . . . 18

3.3 Objective Types and System Characteristics of D2D Resource Allocation Models . . 23 
3.3.1 Single/Multiple Objective . . . . . . . . . . . . . . . . . . . . . . . . 24

3.3.2 Centralized/Distributed Implementation . . . . . . . . . . . . . . . . . . 24

3.3.3 Uplink/Downlink Spectrum Reuse . . . . . . . . . . . . . . . . . . . 26

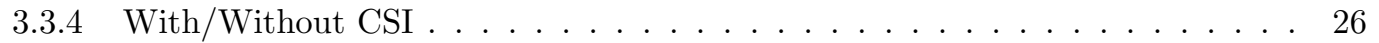

3.4 Tools, Technologies and Applications of D2D Resource Allocation . . . . . . . . . . 28

3.5 Energy Harvesting and Resource Allocation in Underlay D2D Communications . . . 30

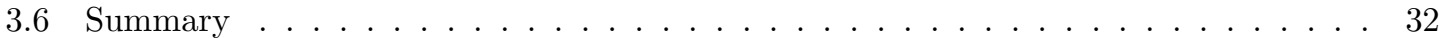

4 Energy Harvesting Assisted D2D Communcations 34

4.1 System Model and Problem Formation . . . . . . . . . . . . . . . . . . . . . . 35

4.1 .1 System Model . . . . . . . . . . . . . . . . . . . . . . . . . . 35

4.1 .2 Problem Formation . . . . . . . . . . . . . . . . . . . . . . . . . . . 39

4.2 OPTI Toolbox and NOMAD Algorithm . . . . . . . . . . . . . . . . 44

4.3 Simulation Results . . . . . . . . . . . . . . . . . . . . . . . 46

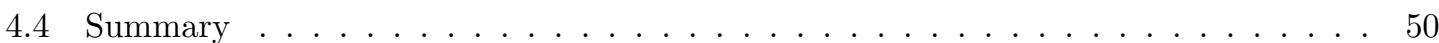

5 A Heuristic RB and Power Allocation Algorithm 51

5.1 Heuristic Algorithm . . . . . . . . . . . . . . . . . . . . . . 51

5.2 Simulation Results . . . . . . . . . . . . . . . . . . . . . . . . 53

5.3 Results Comparison with NOMAD Algorithm . . . . . . . . . . . . . . . 58

5.3.1 Random RB Allocation with Equal Power Distribution . . . . . . . . . . . . . 58

5.3 .2 Percentage of Active D2D Users _ . . . . . . . . . . . . . . . . . 59

5.3 .3 Sum Throughput . . . . . . . . . . . . . . . . . . . . . . . 60

5.3 .4 Time Cost . . . . . . . . . . . . . . . . . . . . . . 61

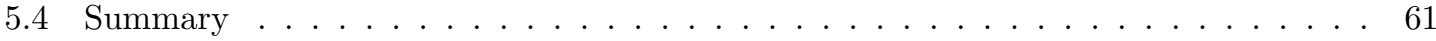

6 Conclusions and Future work $\quad 63$

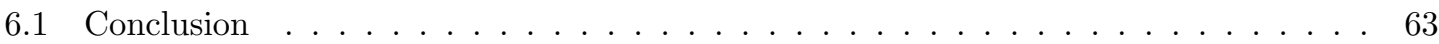

6.2 Future Work . . . . . . . . . . . . . . . . . . . . . 64

$\begin{array}{ll}\text { Bibliography } & 65\end{array}$ 


\section{List of Tables}

2.1 A comparison of different varieties of D2D communications systems . . . . . . . . 10

3.1 Problem types and solutions . . . . . . . . . . . . . . . . . . . . . . 22

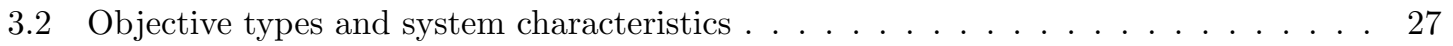

3.3 Energy harvesting in D2D communications $\ldots \ldots \ldots \ldots \ldots \ldots$

4.1 Description of the symbols used in the model. . . . . . . . . . . . . . . . . 41

4.2 Simulation parameters. . . . . . . . . . . . . . . . . . . . 46 


\section{List of Figures}

2.1 Interferences in s typical underlay D2D communications system. $\ldots \ldots \ldots \ldots \ldots$

2.2 A simple categorisation of $\mathrm{D} 2 \mathrm{D}$ communications $\ldots \ldots \ldots \ldots$

2.3 Resource block in LTE networks. . . . . . . . . . . . . . . . . . . . . . . . . . . . 12

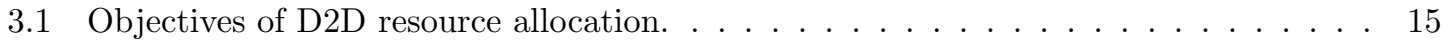

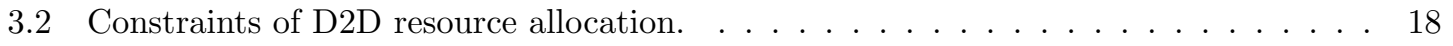

3.3 Tools, technologies and applications of D2D resource allocation. . . . . . . . . . 28

4.1 A simple scenario of system model. . . . . . . . . . . . . . . . . . . . . . 36

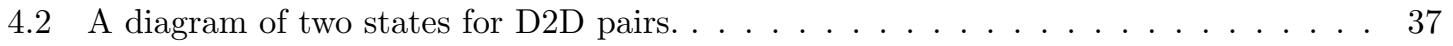

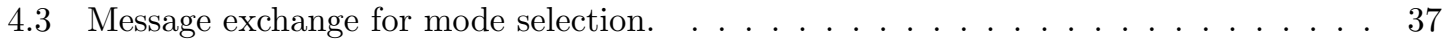

4.4 Relationship between EH efficiency factor and percentage of active D2D pairs. . . . 47

4.5 Sum throughput of D2D users on varying number of CUs. . . . . . . . . . . . 48

4.6 Sum throughput of D2D users on varying QoS requirements for CUs. . . . . . . . . 49

5.1 Relationship between EH efficiency factor and percentage of active D2D pairs. . . . 53

5.2 Total energy harvested on varying number of D2D users. . . . . . . . . . . . . 54

5.3 Sum throughput of D2D users on varying number of D2D users. . . . . . . . . 55

5.4 Sum throughput of D2D users on varying number of CUs. . . . . . . . . . 56

5.5 Sum throughput of D2D users on varying QoS requirements for CUs. . . . . . . . . . 57

5.6 A comparison of average percentage of active D2D users between two algorithms . . 59

5.7 A comparison of D2D sum throughput under different algorithms . . . . . . . . . . 60

5.8 A comparison of time cost under different algorithms . . . . . . . . . . . . . 61 


\title{
List of Abbreviations
}

\author{
D2D Device-to-Device Communications \\ EH Energy Harvesting \\ CU Cellular User \\ BS Base Station \\ EE Energy Efficiency \\ RB Resource Block \\ QoS Quality of Service \\ OFDMA Orthogonal Frequency Division Multiple Access \\ MINLP Mixed Integer Nonlinear Programming \\ NOMAD Nonlinear Optimization by Mesh Adaptive Direct Search \\ WSNs Wireless Sensor Networks \\ HetNets Heterogeneous Networks
}




\section{Chapter 1}

\section{Introduction}

\subsection{Context of the Study}

This thesis deals with the resource allocation problem of device-to-device (D2D) communications in an energy harvesting assisted underlay system. Resource allocation has been the centre topic of D2D communications since it was brought up first in the academia, especially in the underlay D2D system, where the interference between cellular users as primary users and D2D users as secondary users is more likely to compound the resource allocation process to satisfy the QoS requirements for both users. Energy harvesting, another novel technology that has already gained much traction in wireless sensor networks (WSN), is starting to be explored in D2D communications gradually. A few papers in the literature have presented causal or non-causal models of energy harvesting assisted D2D communications.

\subsection{Objective and Contributions of the Thesis}

The objective of this thesis is the maximization of sum throughput for all D2D users underlaying cellular users in energy harvesting assisted OFDMA cellular networks. The summary of our contributions is shown as follows:

i) establishing a unique, centralized energy harvesting assisted D2D model based on the remaining energy level in D2D users, 
ii) formulating an optimization problem of sum throughput based on multiple optimization elements, constraints and assumptions,

iii) providing a mathematic analysis of the optimization problem and solving the problem with the help of NOMAD algorithm in the OPTI toolbox in MATLAB,

iv) offering a heuristic resource allocation algorithm to solve the proposed problem and comparing the results with the ones obtained from the OPTI toolbox.

\subsection{Thesis Organization}

The organization of the thesis is very straightforward. We started with literature review and then segue into system model, problem formulation and finally simulation results and analysis. The detailed summary of each chapter is shown below:

Chapter 2 provides a general overview of D2D communications, which includes the definition, application and system architecture of D2D communications, categorisation and comparison of various D2D communications systems, and some of the most commonly explored resource allocation elements in underlay D2D communications systems in the literature.

Chapter 3 presents a survey of resource allocation schemes in underlay D2D communications following the concept of resource allocation briefly mentioned in the last chapter. We go into details of a collection of papers, discussing several aspects of D2D resource allocation including objectives and constraints, problem types and solutions, objective types and system characteristics, and some tools, technologies and applications of these D2D resource allocation. Analyses of these aspects offer us invaluable insights into how we should construct a generic D2D model based on legitimate assumptions. In the final section, we introduce the concept of energy harvesting into D2D communications based on some recent articles, which provides a foundation for our own energy harvesting assisted D2D system model.

Chapter 4 begins with the construction of such energy harvesting aided D2D system model. We meticulously introduce the two states for D2D users in our system, how the transition between different states works and the rationale behind it, then we derive an optimization problem that is aimed at obtaining maximal sum throughput for D2D users in a certain amount of time slots. In order to solve the problem, we reduce it into instances of generic scenario in one time slot after mathematical analysis and simplification, which turns out to be a MINLP problem. Finally, 
we apply the solver of NOMAD algorithm provided in the OPTI toolbox to produce an optimal solution.

Chapter 5 presents an alternative approach by using heuristic joint resource allocation algorithm to solve the same problem presented in Chapter 4. Since there are limitations on the number of variables in NOMAD solver, we were only able to produce simulation results based on a maximum of $4 \mathrm{D} 2 \mathrm{D}$ pairs using NOMAD algorithm. Our heuristic algorithm is able to generate more results to confirm its efficacy. We then compare some of the results with the ones we obtained in Chapter 4 to offer a more thorough analysis.

Chapter 6 recapitulates the overall contributions of our thesis and sheds light on the directions regarding the future work of this topic. 


\section{Chapter 2}

\section{Introduction of D2D}

\section{Communications and Resource}

\section{Allocation}

Social networking applications are everywhere nowadays; from Facebook to Twitter, from Instagram to Snapchat, all these trendy data-intensive applications are changing our daily lives thanks to the popularity of mobile devices. However, the exponential growth of these devices still cannot fully satiate people's growing need to communicate as more applications providing proximity-based services are emerging in our daily lives. Moreover, 4G cellular technology, which has efficient physical and MAC layer performance, is still lagging behind mobile users' booming data demand. Besides, applications that provide video streaming or file sharing services are draining the already limited bandwidth resources. Therefore, how to realize efficient spectrum utilization is an urgent problem that needs to be dealt with.

Researchers have been seeking for new paradigms to revolutionize the traditional communication methods of cellular networks. Device-to-Device (D2D) communications is one of these paradigms that appears to be a promising component in the next generation cellular technologies. What is D2D communications? In the following sections, we will introduce the definition, architecture and applications of D2D communications, the characteristics and categorisation of communications 
system, and some common resource allocation elements in underlay D2D communications systems.

\subsection{D2D Communications: Definition, Application and Sys- tem Architecture}

Device-to-Device (D2D) communications refers to a type of direct communications between two devices in proximity to each other without travelling through the base station (BS). As a new mode of communications that takes advantage of geographical adjacency, it is investigated as proximity services (ProSe) in the 3rd Generation Partnership Project (3GPP). In fact, there are many similar technologies previously that share such functionality such as Wi-Fi, Bluetooth and Zigbee, etc. The issue is that these technologies all work in unlicensed frequency bands and, therefore, cannot provide enough security and meet the requirement of quality of service (QoS). D2D communications, however, can occur in both cellular spectrum and unlicensed spectrum.

In academia, D2D communication was first proposed to enable multi-hop relays in cellular networks. Later some researchers started investigating the potential of D2D communications for improving spectral efficiency of cellular networks. Other potential D2D use-cases were also introduced in the literature such as multi-casting, peer-to-peer communications, video dissemination, machine-to-machine (M2M) communications, cellular offloading, and so on. The first attempt to implement D2D communication in a cellular network was made by Qualcomm's FlashLinQ [1], which is a PHY/MAC network architecture for D2D communications underlaying cellular networks. FlashLinQ takes advantage of OFDM/OFDMA technologies and distributed scheduling to create an efficient method for timing synchronization, peer discovery, and link management in D2D-enabled cellular networks.

Compared with traditional cellular communications, D2D communications has many advantages. In a traditional cellular network, all communications must traverse through the base station even if both parties are close enough for D2D communication. Such architecture suits the conventional low data rate mobile services such as voice call and text message, in which users are not usually close enough to have direct communication. However, mobile users in today's cellular networks often favour high data rate services such as video streaming, online gaming and dozens of proximity-aware social networks, which could cause severe overload to the system if simply following the approach of traditional cellular communications. We can see why D2D communications can 
be particularly rewarding in such cases. Besides, the advantages of D2D communications are not only limited to enhanced spectral efficiency and higher sum throughput. In addition to improving spectral efficiency, D2D communications can potentially improve energy efficiency, system latency and overall fairness. As the demand for higher-data-rate applications keeps snowballing, we have enough reasons to believe that D2D communications will play a major role in the $5 \mathrm{G}$ communications in order to help unload the burden off cellular networks and achieve better quality of service (QoS) performance for all users of interest.

The applications of D2D communications are very encompassing. Other than offloading the traffic of traditional cellular networks, which is the primary function since its introduction, D2D communications can be applicable to many exciting services. First of all, they can bring about more proximity-based social applications that involve device discovery and connection building because such functionality would reduce the cost of communication between devices. Local vendors can send promotion messages via D2D communications to their potential customers more efficiently and almost spontaneously. Medical staff can reach areas where traditional networks might be compromised in case of natural disasters such as fires and earthquakes by exploiting D2D communications. Even dating services can be made more accessible and more feasible if we apply the magic of D2D communications properly.

In addition, it is advantageous to have an alternative method that can serve as a supplementary means of communication for the public to use for sharing information such as text transmission, file sharing, real-time audio and/or video streaming to each other, etc while at the same time avoid causing too much traffic to the cellular network, which is actually closely associated with the development of social networking. In the fifth generation $(5 \mathrm{G})$ wireless communication, the application scenarios can be even more abundant with the availability of higher data rates and capacity.

D2D communications can change our lives tremendously through these amazing applications. We can share files and media data with our friends nearby almost at any time without worrying about the condition of current network and the overconsumption of mobile data. Therefore, a lot of new features must be added on to the current mobile social networking services. Also, when we pass by a local restaurant or a grocery store, we can get free message of latest coupons and promotion information in no time. We can also choose to block the message if we think it may disturb us. All of these must be attributed to the flexibility and efficiency that otherwise cannot 

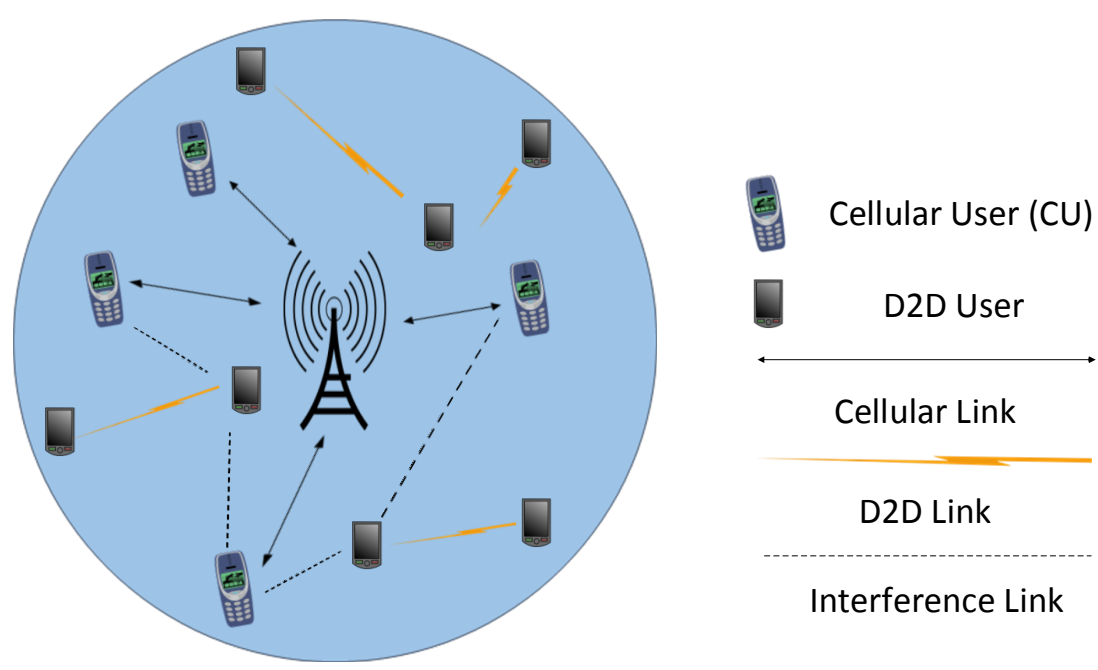

Figure 2.1: Interferences in s typical underlay D2D communications system.

be consummated. When emergencies or disasters occur, the use of D2D communications can offer us a better chance of rescuing ourselves and helping others. The list can go on and on; we could never imagine how much impact D2D communications can bring to our lives.

However, opportunity usually presents itself along with challenges. One of the biggest challenges in D2D communications right now lies in its extremely complicated interference scenarios. The interferences in a typical D2D communications system can be divided into two parts in general: intra-cell interference and inter-cell interference. The former is decidedly stronger, however, the latter is far from negligible when we are expecting a reliable and accurate system. Since most of the times, D2D communications functions as an underlay of the cellular networks, reusing the same resources of the cellular users, the interference level can be further amplified. Fig. 2.1 demonstrates how interference in a D2D communications networks can affect the QoS of cellular users. The interference will inevitably affect the performance or even jeopardize the basic function of D2D communications if not properly handled. Therefore, we need effective resource allocation techniques to facilitate smooth operation of D2D communications along with the cellular users. 


\subsection{Categorisation and Comparison of D2D Communications Systems}

In this section, we will briefly discuss the categorisation of D2D communications systems and offer a thorough comparison of advantages and disadvantages in different types of D2D communications systems before delving into the resource allocation elements of D2D communications as we believe these discussions are necessary to the clear understanding of resource allocation in D2D communications.

As we have mentioned earlier, D2D communications can happen in both cellular spectrum and unlicensed spectrum. Based on the spectrum it utilizes, we can divide D2D communications into two major categories: inband D2D communications and outband D2D communications as shown in Fig. 2.2.

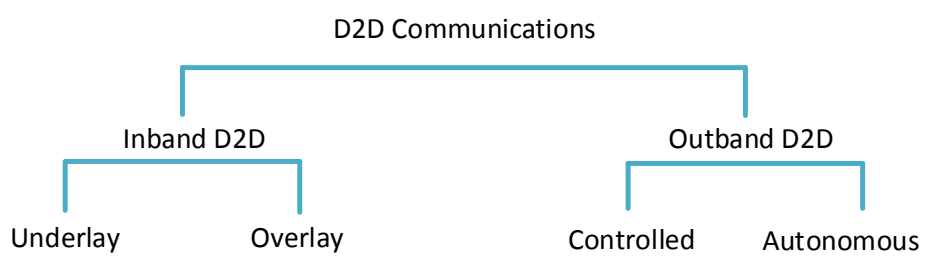

Figure 2.2: A simple categorisation of D2D communications

Inband D2D communications can be further divided into underlay and overlay categories. In underlay D2D communications, cellular users and D2D pairs share the same radio resources. In contrast, D2D pairs in overlay D2D communications are given dedicated cellular resources. Inband D2D communications can improve the spectrum efficiency of cellular networks by reusing spectrum resources or allocating dedicated cellular resources to D2D users that will form a direct connection between the transmitter and the receiver. One of the major disadvantages for inband D2D communications is due to the interference caused by spectrum sharing. However, the interference can be mitigated with more complex resource allocation schemes at the cost of huge computational overhead for both the BS and D2D users.

Outband D2D communications, on the other hand, exploits unlicensed spectrum. The moti- 
vation behind using unlicensed spectrum is to eliminate the interference between D2D links and cellular links. Unlicensed spectrum, however, requires an extra interface and usually adopts other wireless technologies such as WiFi Direct, ZigBee, Bluetooth, etc. Another downside of outband D2D communications is that D2D users may suffer from the capricious nature of unlicensed spectrum. Since only cellular devices with two wireless interfaces can use outband D2D communications, for example, LTE and WiFi, users of these devices can have cellular communications and D2D communications at the same time.

Outband D2D communications can also be divided into two categories: controlled if it is managed by the cellular, and autonomous if it operates on its own. The aim of controlled D2D communications is usually to improve system performance in terms of sum throughput, energy efficiency, etc. while autonomous D2D communications is often motivated by reducing the overhead of cellular networks. In general, works regarding outband D2D communications are deficient.

In the following, we will discuss the advantages and disadvantages of each type of D2D communications individually and offer a thorough comparison of inband D2D communications and outband D2D communications to summarize the similarities and differences with each other.

The advantages of inband D2D communications include:

- underlay D2D increases the spectral efficiency of cellular spectrum by exploiting the spatial diversity;

- almost any cellular device is capable of using inband D2D as compared to outband D2D which is not supported by most cellular devices;

- QoS management is easy because the cellular spectrum can be fully controlled by the BS.

The disadvantages of inband D2D communications include:

- inband D2D is unable to take advantage of the extra cellular resources in overlay D2D;

- interference management in underlay communications is more complicated and challenging for both D2D users and cellular users;

- one user cannot have both D2D communications and cellular communications at the same time.

The advantages of outband D2D communications include: 
- there is no interference between cellular and D2D users;

- resource allocation becomes easier because the scheduler does not require to take the frequency, time, and location of the users into account;

- simultaneous D2D and cellular communication is feasible.

The disadvantages of outband D2D communications include:

- interference in unlicensed spectrum is not in the control of the BS;

- only cellular devices with two radio interfaces (e.g., LTE and WiFi) can take advantage of outband D2D;

- power control between two wireless interfaces is more critical to lower the overall power consumption of the device.

Table 2.1: A comparison of different varieties of D2D communications systems

\begin{tabular}{|l|c|c|c|c|}
\hline \multicolumn{1}{|c|}{ Spectrum Utility } & \multicolumn{2}{c|}{ Inband } & \multicolumn{2}{c|}{ Outband } \\
\hline Various Modes & Underlay & Overlay & Controlled & Autonomous \\
\hline Interference between D2D and cellular users & $\checkmark$ & $\times$ & $\times$ & $\times$ \\
\hline Dedicated resources for D2D users needed & $\times$ & $\checkmark$ & $\times$ & $\times$ \\
\hline Controlled interference scenarios & $\checkmark$ & $\checkmark$ & $\times$ & $\times$ \\
\hline Simultaneous D2D and cellular transmission & $\times$ & $\times$ & $\checkmark$ & $\checkmark$ \\
\hline More than one radio interface needed & $\times$ & $\times$ & $\checkmark$ & $\checkmark$ \\
\hline Coordination between platforms needed & $\times$ & $\times$ & $\checkmark$ & $\checkmark$ \\
\hline Increased overhead for scheduler & $\checkmark$ & $\checkmark$ & $\checkmark$ & $\times$ \\
\hline
\end{tabular}

Table 2.1 summarizes the pros and cons of four types of D2D communications. According to the literature, inband underlay D2D communications systems are the most commonly discussed. One reason is that allocating dedicated spectrum resources to D2D users in an overlay system is not as efficient as underlay in terms of spectral efficiency. The other reason is that outband D2D communications has to deal with the capricious nature of unlicensed spectrum. Even though inband underlay D2D communications needs to deal with more interference, it is easier to manipulate as we can control the interference through resource block allocation and power allocation. In the next 
section, we will start the discussion of resource allocation elements in underlay D2D communications systems. In fact, the entire thesis is based on the assumptions of an underlay D2D communications system.

\subsection{Resource Allocation Elements in Underlay D2D Com- munications Systems}

In this section, we provide a list of common resource allocation elements and explain why they are the key problems we need to tackle in the field of D2D communications.

- Mode Selection: In D2D communications underlaying cellular networks, all the scenarios can be generally categorized into two modes among which users can switch due to real-time condition: D2D mode and cellular mode. In the reusing resource mode, D2D users share the same resource with cellular users (CUs) and that will no doubt generate huge mutual interference among D2D users and CUs, and make it more difficult to guarantee the QoS requirements. In the cellular mode, the transmission of D2D users is relayed to the BS when the D2D direct link no longer works as a fallback plan. In this way, BSs can provide ubiquitous access and uniform service for users, which is one of the advantages of D2D communications over other short-range transmission techniques. Both modes have pros and cons, restrictions and merits, which means it is very necessary for us to gain insight into the selection of these modes to avoid or cancel interference as much as possible.

- Resource Block Allocation: In LTE networks, the radio resources are divided into equalsized physical resource blocks or RBs. Each RB occupies 1 time slot (0.5 ms) in time domain and $180 \mathrm{kHz}$ with 12 subcarriers in the frequency domain as shown in Fig. 2.3. Since the amount of interference in each resource block is time- and space-dependent, a better network throughput and spectrum utilization may only be achieved with a discrete selection of the shared resource. Therefore, efficient spectrum resource scheduling is of importance in the research of D2D communications.

- Power Allocation: Power allocation or power control refers to the allocation of power for D2D transmitters as well as CUs during the process of D2D transmission. It is vital in realizing efficient energy usage and interference coordination in cellular networks in that it is 


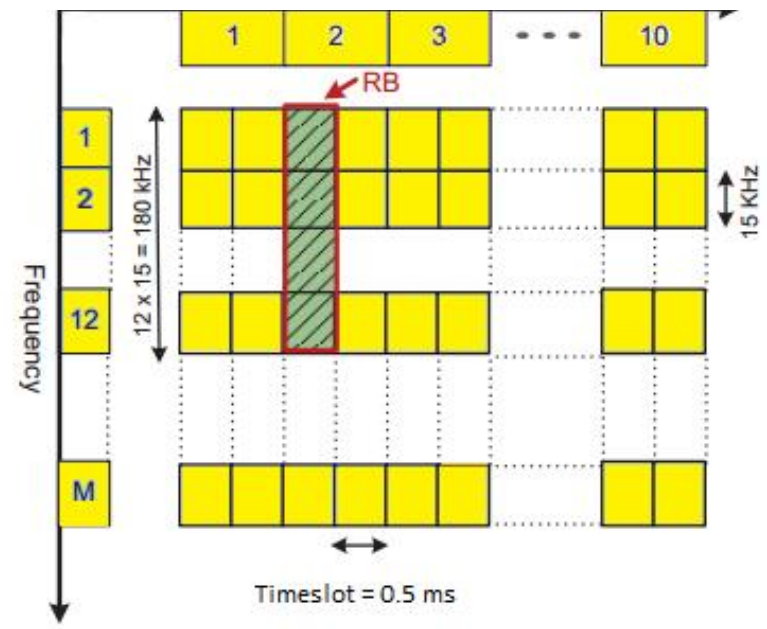

Figure 2.3: Resource block in LTE networks.

a direct way to limit interference. In fact, optimal power allocation is often incorporated in the comprehensive resource allocation algorithm in combination with other resource allocation elements to achieve optimal system performance such as maximal sum throughput or energy efficiency. In D2D communications as an underlay of cellular networks, we usually consider cellular users as the primary users and consider their QoS requirements with priority. Therefore, the first step of power control is to keep the transmission power of D2D pairs in check so that their interference with the CUs does not affect the performance of CUs too much. Also, we may need to consider lowering the power of BS provided that the quality of communications between CUs will not be degraded and the overall performance of the whole network will ameliorate at the same time. In other words, power control should be something considered in a system as a whole instead of being emphasized on one specific part, especially in a D2D communication network where scenarios of interference are even more complicated.

- Rate Control: Rate control or rate adaptation is another critical issue in resource allocation, because it enables a system to adapt to the users rate on itself so that the radio resources can be utilized more efficiently. And that is even more important in D2D communications since the complicated interference scenario in the D2D system requires every possible measurement to constrain interference so that they do not affect each other too much, especially in D2D 
assisted video transmission, where rate control is enabled to encode the video at different target transmission rates for both the D2D link and the cellular link.

Besides the resource allocation elements listed above, there are many other resource allocation elements worth exploring, such as antenna selection, relay selection, etc. Since they are not as general as the ones discussed above, we will not go into details.

\subsection{Summary}

In this chapter, we provided a brief introduction of D2D communications from its definition, history of development, to various potential use cases in our increasingly connected daily lives. We also discussed the system architecture of D2D communications and divided different systems into two major categories, inband D2D and outband D2D, based on the frequency bands used for D2D communications. Inband D2D systems could be further divided into underlay D2D systems and overlay D2D systems. We then compared these different types of systems in detail. Literature review shows that inband underlay is the most common system for D2D communications, so we spent the last section discussing the most typical resource allocation elements in underlay D2D communications systems.

In the next chapter, we will provide a comprehensive survey of resource allocation schemes in underlay D2D communications. We will cover the objectives and constraints of a wide range of D2D resource allocation problems, the nature of those problems and their algorithmic solutions, assumptions and characteristics of their system models, tools and technologies related to solve the problems, and miscellaneous applications of D2D resource allocation. Finally, we will focus on the emerging technology of energy harvesting and how it can help us better allocate important resources in underlay D2D communications. 


\section{Chapter 3}

\section{Survey of Resource Allocation Schemes in Underlay D2D communications}

Resource allocation is at the core of D2D communications, as we learned from last chapter. How to allocate a multitude of resource elements can significantly affect how well the systems perform, especially in underlay D2D communications.

In this chapter, we provide a comprehensive literature review of resource allocation schemes in underlay D2D communications. In the first parts of this chapter, we discuss various aspects of the resource allocation problem in a generic D2D communications system. In the final part of this chapter, we focus on energy harvesting assisted D2D communications, including advantages and challenges that are unique to energy harvesting (EH) in D2D communications and how optimal resource allocation techniques are developed to address them. This part also serves as the technical foundation for the work in our thesis as we draw many assumptions and inspirations from the extant EH-aided underlay D2D models. 


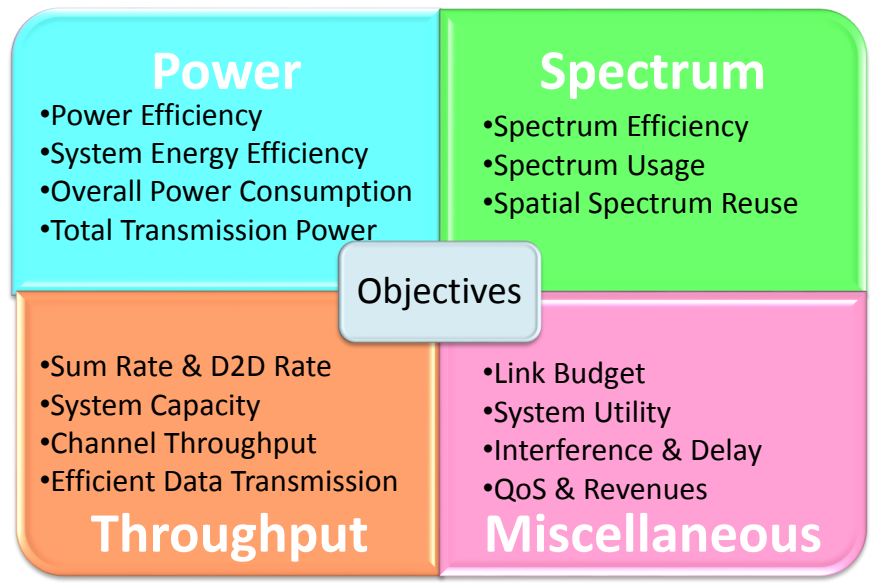

Figure 3.1: Objectives of D2D resource allocation.

\subsection{Objectives and Constraints of D2D Resource Allocation}

In this section, we categorize the objectives and constraints of the resource allocation problems available in the literature into several aspects respectively. To begin with, we concentrate on the objectives of $\mathrm{D} 2 \mathrm{D}$ resource allocation. These objectives can be divided into four categories at large: power, spectrum, throughput and a group of miscellaneous purposes as shown in Fig. 3.1.

A. Power: One of the most common objectives of resource allocation in D2D communications based on literature review is about power. Power allocation is, of course, the most essential element in resource allocation for D2D communications. Since the mobile devices are growing exponentially on a daily basis and carbon emission has turned climate change into a problem that we cannot afford to neglect anymore, therefore, how to allocate and utilize power efficiently should call for everyone's attention now. Besides, operators would like to save expense and cut cost as much as they could, so effective power control is as paramount to the future of their business as to the future of our environment. In [2], the authors put forward an evolutionary approach of mode selection between cellular mode and D2D mode in cognitive networks $(\mathrm{CNs})$ to maximize power efficiency. In [3], the authors focused on minimization problem of total transmit power by combining how to utilize radio resource and power efficiently altogether. Similarly, a joint problem of mode selection, modulation- 
and-coding scheme (MCS) assignment, resource allocation and power control was discussed in [4] to achieve minimal overall power consumption. In [5-7], authors aimed to maximize solely the system energy efficiency while in [8] the authors attempt to maximize both system energy efficiency and energy efficiency for individual D2D users.

B. Spectrum: Another important theme in D2D resource allocation is to realize efficient spectrum usage. As we know, spectrum resource is very scarce, and a large portion of them has already been occupied and utilized nowadays, however, at the same time the demand of higher data rate and the increase of mobile devices are rather unstoppable. Caught up in such a dilemma, we are pressured to come up with new ideas to take advantage of the limited resources efficiently through either proper spectrum allocation strategies or spectrum reuse schemes. In [9], the authors proposed a promising approach for D2D transmissions to serve the intra-cell traffic load through uplink spatial spectrum reuse in LTE. In [10], the authors came up with a novel strategy for spectrum usage in LTE-A based on the theory of ant colony optimization (ACO) after constructing a graph of interference among each D2D user. In [11], a spectrum allocation problem for full duplex cellular networks was discussed. By properly choosing D2D users to help offload traffic off the main cellular networks, the algorithm effectively enhances the spectrum efficiency by providing services to more user equipment with limited spectrum resources.

C. Throughput: Most of the papers in the literature focus on maximizing throughput or data rate one way or another. Throughput is irrefutably one of the important aspects when it comes to determine the efficacy of a system. In fact, how to provide high throughput at low cost is the question throughout the entire development of telecommunications industry. In $5 \mathrm{G}$ communications systems, the peak data rate is supposed to be $10 \mathrm{Gbps}$ and the network capacity is supposed to be 10,000 times the current capacity in order to support the abundance of devices. Also, all operators are wrecking their brains to be the top player in this billowing battle of who can provide the fastest speed of cellular transmission. Therefore, we are poised to believe that throughput is the core in D2D communications as well. In [12-16], the authors concentrated on maximizing sum rate of both cellular users and D2D users while authors in [17-21] focused specifically on the maximization of the data rate for D2D users. In [22], the authors took into account a concept called sum rate gain, which refers to the increased amount of sum rate after the repeated game. In [23], a Kuhn-Munkres based resource allocation algorithm was discussed to maximize the channel throughout for intra-cluster D2D communications. 
D. Miscellaneous: Besides the aforementioned three main genres of objectives, there remain many miscellaneous purposes for resource allocation. In this part, the optimization factors include link budget, system utility, interference, delay, QoS, revenues, etc. In [24], the authors proposed a centralized resource allocation technique that focuses on enhancing the link budget gains and thus size of BS coverage. In [25], the authors come up with two algorithms of resource allocation for D2D communications to minimize the consumption of cellular resources in vehicular ad hoc networks (VANETs). Authors in [26] chose delay as their main optimization element by proposing a flexible D2D concept based on flexible UL/DL TDD scheduling. Simulations show that the said approach can improve overall system performance in terms of decreased package delay and increased throughput. In [27], the authors introduced a concept of 'utility' and 'utility gain' for voice users and data users as a metric of system performance. The BS delegates resource blocks (RBs) based on the largest utility gain during every iteration in order to accommodate more users at the same time. In [28], the authors proposed a QoS-aware resource allocation technique for D2D video streaming. Since video streaming requires stable and smooth performance, the authors put extra emphasis on guaranteeing QoS.

After an anatomy of the objectives for D2D resource allocation, we now move on to the discussion of constraints in D2D resource allocation. Fig. 3.2 lists some common constraints in D2D resource allocation problems. Power definitely accounts for one of the major part of constraints, especially the transmission power, because from the perspective of energy efficiency, we need to set up an upper bound on the level of transmission power or sub-channel power allowed in the system. Second, as higher transmission power often leads to higher interference, especially in D2D communications as an underlay, where we do not want the operation of D2D users eclipse the performance of cellular users, many authors take interference into account as one of the key constraints in their problem formation as well. Another common component of constraints is based on throughput. Usually in D2D communications, there is a minimum data rate or capacity requirement. If this minimum rate cannot be satisfied, then apparently D2D mode is not a remunerative option in this case for the potential users. Finally, there are limitations on the available radio resource in terms of number of RBs or number of sub-channels depending on different multiple access techniques. In the literature, the authors often consider a combination of different constraints. The most common ones are shown in $[5,6,8,13,21,29-31]$, where the authors empathize maximum transmission power and minimum data rate requirements for D2D users as their system constraints. In [15,32-35], interference was 


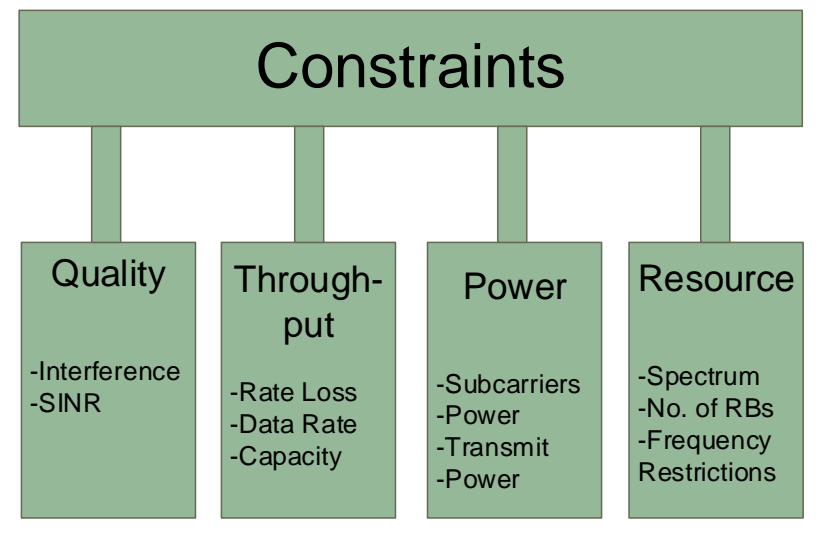

Figure 3.2: Constraints of D2D resource allocation.

also considered by the authors to satisfy QoS. In [4], the authors added the numbers of RBs as one of the constraints in their radio allocation scheme. Similarly, the authors in $[19,20]$ also focus more on frequency constraints in order to maximize the utilization of spectrum resource.

Our work focuses on the optimization of sum throughput, like proposed by many papers in the literature, with constraints ranging from limited transmit power, QoS requirements for cellular users, interference and the restrictions of resource block number and sharing policy.

\subsection{Problem Types and Solutions of D2D Resource Alloca- tion}

In this section, we will discuss the D2D resource allocation problems in detail by taxonomy of their types and solutions. The most common problem types include non-linear mixed integer programming $[7,19,36,37]$, NP-hard problem [30,38,39], non-convex optimization problem [6, 12] and convex optimization problem [21], etc. In $[5,7,8]$, the authors formulated their problems based on fractional programming theory. In [40], an NP-complete mixed integer linear problem was derived. Each problem has its own way of solution. Therefore, in the following part, we will take a 
closer look at some of the papers that are typical of their types individually.

In [18], a novel bandwidth allocation scheme was proposed by Zhang et al. to maximize the utilities for both D2D users and cellular users while keeping the interference between the two as minimal as possible. In this paper, the authors only consider the reuse of uplink resources in a single-cell scenario because frequency division duplex (FDD) in the downlink is not quite feasible and in comparison the uplink resources obviously have more potential for exploitation. First, they formulate an optimization problem of utility for D2D users under a constraint of a threshold value of cellular utility loss, meaning that the interference imposed by the D2D users on the cellular users should not be too strong. The objective function is shown in (3.1):

$$
\max \sum_{i \in S_{D 2 D}} U_{D 2 D}\left(\sum_{j \in S_{C}} x(i, j) r_{D 2 D}(i, j)\right)
$$

where $r_{D 2 D}(i, j)$ refers to the spectrum efficiency of the $i$-th D2D pair sharing the resource of the $j$-th cellular user and $x(i, j)$ is defined as in $(3.2)$ :

$$
x(i, j)= \begin{cases}1, & \text { if D2D user } i \text { reuses } \mathrm{CU} j^{\prime} s \text { resource } \\ 0, & \text { otherwise. }\end{cases}
$$

The problem is a binary integer programming problem and cannot be solved directly as the computing complexity is very high. Therefore, to solve it, the authors first divide it into smaller ones so that they can tackle each of them later individually through primal-dual decomposition. Then they use the projected gradient method to solve the dual problems by developing a distributed algorithm. Simulation results show that it outperforms the centralized approach in terms of utility especially in scenario of large number of D2D users.

In [36], Cai et al. proposed a capacity oriented resource allocation (CORAL) algorithm that allows a D2D pair to share the resource of multiple cellular users. The objective is to maximize the sum rate of the entire system as show in (3.3)-(3.5).

$$
\begin{aligned}
R_{c} & =\sum_{C_{i} \in \boldsymbol{C}_{S}^{\prime}} \pi_{i j} \times \log _{2}\left(1+\gamma_{i}^{\prime}\right) \\
& +\sum_{C_{i} \in \boldsymbol{C}_{\boldsymbol{S}}} \sum_{D_{i} \in \boldsymbol{D}} \pi_{i j} \times \log _{2}\left(1+\gamma_{i}\right) \\
R_{d} & =\sum_{D_{j} \in \boldsymbol{D}} \sum_{C_{i} \in \boldsymbol{C}_{\boldsymbol{S}}} \pi_{i j} \times \log _{2}\left(1+\gamma_{i}\right)
\end{aligned}
$$




$$
\max \left(R_{c}+R_{d}\right)
$$

where $R_{c}$ and $R_{d}$ refer to the sum rate of cellular users and D2D users respectively and $\pi_{i j}$ indicates whether cellular user $C_{i}$ chooses to share its resource with D2D user $D_{j}$ or not. $\gamma_{i}$ refers to the SINR of cellular user $C_{i}$ if it choose to share its resource while $\gamma_{i}^{\prime}$ refers to the SINR of cellular user $C_{i}$ if it choose not to share its resource.

Since the above problem is a non-linear mixed integer programming problem, traditional approach to solve this problem is in vain. Therefore, the authors develop their own heuristic CORAL algorithm. This approach can be divided into two phases. In the first phase, each D2D user chooses one optimal cellular user for resource reuse. In the second phase, those cellular users whose resources remain yet shared will choose an optimal D2D user to share their resources with. The proposed approach can achieve higher sum rate and lower data loss compared with greedy sum-rate maximization algorithm and conventional random resource allocation algorithm.

In [31], Wu et al. explored the cognitive radio based multicast service with D2D communications. In each multicast group, D2D users can reuse the orthogonal cellular resources for multicast transmission. The objective is to achieve a maximal system capacity under the constraints of SINR and transmission power, as shown in (3.6).

$$
\max B_{i} \cdot\left(\left(1+\frac{P_{i} G_{i}}{\sigma^{2}+P_{j} G_{j}}\right) \cdot\left(1+\frac{P_{j} G_{j d_{w}}}{\sigma^{2}+P_{i} G_{i d_{w}}}\right)^{M}\right)
$$

where $B_{i}$ refers to the bandwidth for cellular user $P U_{i}, P_{i}$ and $P_{j}$ refer to the transmission power for cellular user $P U_{i}$ and D2D user $S U_{j}$ respectively, and $G_{i}$ and $G_{j}$ refer to the channel gain and interference respectively.

To achieve optimal system capacity, optimal power control strategy is needed. The proposed algorithm consists of two steps. In the first step, the authors focused on channel allocation based on minimal interference. In the second step, they formed the power allocation problem as a non-linear programming problem. To solve this problem, the authors introduced several lemmas to prove that the optimal power is equal to the maximal power in the feasible region. Experiment results corroborate the authors' conclusion.

In [10], Liotou et al. proposed a new spectrum allocation scheme based on ACO theory. The system model was built in LTE-A networks and multiple D2D users can reuse the same resource block. The authors first constructed a graph to represent all of the D2D interferences. The optimization 
problem was then expressed as the minimization of summed weights as shown in (3.7).

$$
\min \sum_{1}^{L_{l e n}} W\left(v_{i}, v_{j}\right), \text { where } v_{i}, v_{j} \in V .
$$

After the formation of this expression, the authors further apply ACO theory to solve the problem. Simulation results confirm that the said approach can converge fast to spectrum ratio, which could not have been possible by traditional spectrum allocation approaches. However, user mobility is not discussed in this paper and power control is also missing in the paper. In [8], a resource allocation scheme is proposed by Hoang et al. in order to optimize two energy-related metrics: system energy efficiency (SEE) and total individual energy efficiency (TIEE). These two metrics can be expressed as the formula in (3.8) and (3.9).

$$
\begin{gathered}
\max \operatorname{SEE}(\boldsymbol{p}, \rho)=\sum_{l \in K_{D}} \sum_{k \in K_{C}} \rho_{l}^{(k)} r_{l}^{(k)} \\
\max \operatorname{TIEE}(\boldsymbol{p}, \rho)=\sum_{l \in K_{D}} \frac{\sum_{k \in K_{C}} \rho_{l}^{(k)} r_{l}^{(k)}}{p_{l}^{\text {total }}},
\end{gathered}
$$

where SEE represents the overall system energy efficiency while TIEE represents the total individual energy efficiency. $\rho_{l}^{(k)}$ is equal to 1 if channel $k$ for cellular link $k$ is also allocated to D2D link l, otherwise it is equal to $0 . r_{l}^{(k)}$ refers to the data rate of D2D link l over channel $k$.

Both the SEE problem and the TIEE problem are fractional programming problems. In order to solve the SEE problem, the authors first applied the Dinkelbach algorithm to transform it into a non-linear mixed integer programming problem. After that, they further simplify the problem into linear integer programming problem by optimal power allocation so they can then solve it by applying the Hungarian algorithm for channel allocation. As to the TIEE problem, they solved it in a similar manner. First they applied an optimal power allocation technique for TIEE, then the Hungarian algorithm for channel allocation. The only difference lies in the way they choose to simplify the problem through optimal power allocation. Simulation results show that said approach can lead to convergence faster for both metrics. Besides, compared with other approaches, the said approach can achieve a better maximization of spectrum efficiency.

In [7], a resource allocation problem for D2D overlaying LTE networks was discussed. Overlay means that D2D users and cellular users both have dedicated orthogonal spectrum resources instead of sharing the same one. In this case, the interference is relatively smaller. However, different 
D2D users might share orthogonal or non-orthogonal spectrum resource. The resource allocation problems for both two scenarios are firstly formulated as a fractional programming (FP) problem and a non-linear mixed integer fractional programming problem respectively whose goals are to maximize energy efficiency. Both of them can then be simplified into equivalent subtractive form in terms of parameters based on fractional programming theory. The simplified problems are nonconcave, so the authors come up with a heuristic scheme based on Dinkelbach and Powell-HestenesRockafellar augmented Lagrangian methods. Numerical results further demonstrate that the said approach can achieve higher energy efficiency and can perform even better during orthogonal mode than non-orthogonal mode.

Besides the above examples, there remain many papers which take similar or different approaches to solve similar problems. Table 3.1 gives a brief summary of problem types and solution approaches mentioned in a dozen of papers.

Table 3.1: Problem types and solutions

\begin{tabular}{|l|l|l|}
\hline Ref. & Problem Type & \multicolumn{1}{c|}{ Solution Approach } \\
\hline$[5]$ & Fractional non-convex problem & $\begin{array}{l}\text { Dual problem formulation and fractional program- } \\
\text { ming }\end{array}$ \\
\hline$[6]$ & Non-convex problem & $\begin{array}{l}\text { Constraint relaxation into non-linear fractional pro- } \\
\text { gramming solved by Dinkelbach }\end{array}$ \\
\hline$[7]$ & $\begin{array}{l}\text { Fractional programming problem; } \\
\text { Mixed integer non-linear fractional } \\
\text { programming }\end{array}$ & $\begin{array}{l}\text { Non-concave equivalent problems solved by Dinkel- } \\
\text { bach and Powell-Hestenes-Rockafellar augmented } \\
\text { Lagrangian methods }\end{array}$ \\
\hline$[8]$ & Fractional programming & $\begin{array}{l}\text { Dinkelbach-based algorithm and Hungarian method } \\
\text { Heuristic algorithm based on ant colony optimization } \\
\text { (ACO) }\end{array}$ \\
\hline$[10]$ & Interference-based graph problem & $\begin{array}{l}\text { Hungarian algorithm for channel assignment and } \\
\text { greedy algorithm for data offloading }\end{array}$ \\
\hline$[18]$ & Bipartite graph problem & $\begin{array}{l}\text { Subgradient-based algorithm by applying La- } \\
\text { grangian dual theory }\end{array}$ \\
\hline
\end{tabular}




\begin{tabular}{|c|c|c|}
\hline \multicolumn{3}{|c|}{ Continuation of Table 3.1} \\
\hline Ref. & Problem Type & Solution Approach \\
\hline$[19]$ & $\begin{array}{l}\text { Non-linear mixed integer program- } \\
\text { ming }\end{array}$ & $\begin{array}{l}\text { An iterative algorithm to decouple the original prob- } \\
\text { lem into two sub allocation problems }\end{array}$ \\
\hline$[21]$ & Convex problem & Lagrangian multipliers with KKT conditions \\
\hline$[30]$ & NP-hard problem & $\begin{array}{l}\text { Interference graph-based allocation (InGRA) algo- } \\
\text { rithm }\end{array}$ \\
\hline$[31]$ & Non-linear optimization & Heuristic algorithm for power allocation problem \\
\hline$[35]$ & NP-hard problem & $\begin{array}{l}\text { Greedy Throughput Maximization Plus (GTM+) al- } \\
\text { gorithm }\end{array}$ \\
\hline$[36]$ & $\begin{array}{l}\text { Non-linear mixed integer program- } \\
\text { ming }\end{array}$ & $\begin{array}{l}\text { Capacity-oriented resource allocation (CORAL) al- } \\
\text { gorithm }\end{array}$ \\
\hline$[37]$ & $\begin{array}{l}\text { Non-linear mixed integer program- } \\
\text { ming }\end{array}$ & Fractional knapsack with greedy algorithm \\
\hline$[38]$ & NP-hard problem & $\begin{array}{l}\text { Heuristic algorithm based on graph coloring-based } \\
\text { model }\end{array}$ \\
\hline$[39]$ & NP-hard problem & Novel interference-aware bipartite graph \\
\hline$[40]$ & $\begin{array}{l}\text { NP-complete mixed integer linear } \\
\text { problem }\end{array}$ & $\begin{array}{l}\text { Near-optimal CLIP (clustering and iterative linear } \\
\text { programming) algorithm }\end{array}$ \\
\hline \multicolumn{3}{|c|}{ End of Table } \\
\hline
\end{tabular}

\subsection{Objective Types and System Characteristics of D2D Re- source Allocation Models}

In this section, we will discuss about the objective types and system characteristics of a series of D2D resource allocation models proposed by various authors. A thorough categorization of these attributes about system modelling, problem formation, objectives and implementation will help readers understand clearly the trend of solutions for D2D resource allocation problems. We will figure out which ideas have been thoroughly explored and which ones assume the potential for further research. 
All D2D system models used in resource allocations are similar one way or another, but none are exactly the same. In order to have a clear idea of the progress in the academia regarding this topic, we have decided to explore the system characteristics of D2D models presented in the literature through a series of classification in this section.

We can categorize the system models with the following characteristics: single or multiple objectives, centralized or distributed implementation, uplink or downlink spectrum reuse, with or without channel state information(CSI).

\subsubsection{Single/Multiple Objective}

To start with, we have noticed that most papers that we reviewed form an optimization problem that is single-objective, dealing with either the maximization of sum data rate or the minimization of energy consumption, but authors in few papers [17] and [13] proposed a multiple-objective optimization problem.

In [17], a multi-objective utility function was proposed for both the cellular and D2D users in the formation of Stackelberg game. The base station acts as a leader who co-ordinates interference between cellular users and D2D users while the D2D users assume the role of followers, competing for the limited spectrum to maximize their individual data rates. Mathematical proof shows that such a mechanism will eventually lead to equilibrium where the optimization of spectrum allocation is achieved. In [13], the authors proposed a distributed solution for radio resource allocation in network-assisted D2D communications system. The objective is to maximize the data rates and to satisfy total power consumption requirements for both cellular users and D2D users. Relay nodes are deployed to pass message in this approach. Based on an analysis of objective types, we believe more efforts and energy should be put into the formation of a multi-objective problem regarding D2D resource allocation in order to achieve a balance between QoS and energy efficiency or spectrum efficiency. Multi-objective approaches also tend to have higher fairness than single-objective ones.

\subsubsection{Centralized/Distributed Implementation}

Centralized approaches require the BS to function as a leader, delegating tasks or in this area the power or spectrum resources. It usually entails the access to full control of the system on the BS's behalf; therefore it is not very efficient as extra control messages are needed. In a few 
papers $[6,12,24,33]$, the authors proposed centralized resource allocation schemes.

In [12], the authors attempted to achieve a maximal sum throughput for D2D users through a centralized approach. In this system, each sub-carrier can only be allocated to one D2D user and a D2D link can only utilize this sub-carrier if reusing it can yield a gain above a threshold. To add to that, rate loss of cellular users is also considered in order to guarantee QoS. They then solve it through the application of Lagrangian theory, which proves to be more efficient than the previous water-filling algorithm.

In [24], a D2D voice broadcast model was discussed. The authors then propose a centralized heuristic approach to allocate RBs properly. The said approach can produce a $3-5 \mathrm{~dB}$ gains in terms of link budget, meaning that it can cover larger area.

D2D communications is a promising technique in LTE-A, however, the interference among different D2D users can be intense. That is why in [33], the authors proposed a centralized resource allocation scheme that is interference-aware. They first represented the interference relationships among different D2D users with an interference graph. Then they attempted to allocate the resource by minimizing the conflicts, thus achieving the optimal performance. Other than the above centralized approaches, the majority of the papers have adopted distributed approaches as we can see from the Table 3.2.

Hybrid resource allocation approach is a mixed type of both strategies. Though it is relatively rare in the literature, we do find one example [34]. In this paper, multiple D2D users share the radio resource with the cellular users. In fact, there is no limit on the number of coexisting D2D users with cellular users in the system. The BS has only knowledge of the geographical locations of cellular users and D2D users while both cellular and D2D users have no channel knowledge at all. The authors decoupled the problem into two sub-problems: centralized channel allocation and distributed power control for D2D users. To solve the channel allocation, they applied graph theory, while to solve the power control, they modeled it as a multi-agent learning game. The hybrid approach demonstrates the feasibility in a large-scale area following multiple criteria of appraisal.

In conclusion, distributed approaches are more popular because centralized approaches entail the full control of the system at the BS's end, which is not very easy to actualize. Another advantage of distributed approaches is low complexity, since the burden of decision making and resource allocation process is shared across users. 


\subsubsection{Uplink/Downlink Spectrum Reuse}

Uplink and downlink resource reuse is another essential aspect regarding the system models in the papers. Most papers set up their system models assuming that D2D communications utilizes the uplink spectrum resource only, while a few papers $[30,34,41,42]$ set up their model in the downlink section. For instance, the authors in [42] consider a joint non-orthogonal spectrum sharing problem in the downlink direction. First, they propose an NP-hard problem, and then they employ an iterative algorithm that deals with both spectrum allocation and power allocation during every iteration. Simulation results demonstrate that this non-orthogonal approach performs better than the other orthogonal ones.

In [11], both uplink and downlink D2D resource allocation problems were considered. In this particular scenario, a full duplex-enabled BS is deployed at the center of a full duplex cell, providing service to half duplex UE. To maximize sum throughput, the allocation of resources take place in two steps. In the first step, channel assignment is done in a way to utilize all viable cellular resources through Hungarian algorithm. In the second step, D2D users are brought into the system as relay structures through a greedy selection algorithm to offload cellular traffic and lessen the co-channel interference between the downlink and uplink cellular users due to the full duplex transmission mode.

\subsubsection{With/Without CSI}

Channel State Information (CSI) refers to the information about the properties of the transmission channels. It is often assumed to be known by the BS or some other central controllers in a communications system for purposes of resource allocation in some system models. But in reality not all systems have the ability to build and maintain this table of information on the BS's side. That is why we decide to check its existence in the assumptions of the system models in all the pertinent papers that we could gather. In some papers $[15,17,34]$, CSI is needed for the proposed resource allocation schemes and algorithms. But in many papers $[3,12,14,30,31]$, CSI is not needed or mentioned.

In our energy harvesting assisted model, the system is a centralized one with only downlink spectrum reuse as distributed systems and uplink spectrum reuse models have already been investigated intensively. Base station serves as both a relay for cellular communications and an energy 
Table 3.2: Objective types and system characteristics

\begin{tabular}{|c|c|c|c|c|}
\hline & $\begin{array}{c}\text { Single / Multiple } \\
\text { Objective(s) }\end{array}$ & $\begin{array}{c}\text { Centralized / } \\
\text { Distributed }\end{array}$ & CSI & $\begin{array}{l}\text { Uplink / } \\
\text { Downlink }\end{array}$ \\
\hline Reference & $\mathrm{S} / \mathrm{M}$ & C/D & $\mathbf{Y} / \mathbf{N}$ & $\mathrm{U} / \mathrm{D}$ \\
\hline$[2]$ & S & $\mathrm{D}$ & & $\mathrm{U}$ \\
\hline$[3]$ & S & & $\mathrm{Y}$ & \\
\hline$[6]$ & S & $\mathrm{C}$ & & \\
\hline$[11]$ & S & $\mathrm{D}$ & & Both \\
\hline$[12]$ & S & $\mathrm{C}$ & $\mathrm{Y}$ & \\
\hline$[13]$ & M & $\mathrm{D}$ & & \\
\hline$[14]$ & S & & $\mathrm{Y}$ & U \\
\hline$[15]$ & S & & & \\
\hline$[16]$ & S & & & $\mathrm{U}$ \\
\hline$[17]$ & M & D & & U \\
\hline$[18]$ & $\mathrm{S}$ & D & & U \\
\hline$[21]$ & S & $\mathrm{D}$ & & \\
\hline$[24]$ & S & $\mathrm{C}$ & & \\
\hline$[27]$ & $\mathrm{S}$ & & & $\mathrm{U}$ \\
\hline$[29]$ & S & $\mathrm{D}$ & & \\
\hline$[30]$ & S & & $\mathrm{Y}$ & $\mathrm{D}$ \\
\hline$[31]$ & $\mathrm{S}$ & & $\mathrm{Y}$ & U \\
\hline$[33]$ & $\mathrm{S}$ & $\mathrm{C}$ & & $\mathrm{U}$ \\
\hline$[34]$ & $\mathrm{S}$ & Hybrid & & $\mathrm{D}$ \\
\hline$[40]$ & S & & & $\mathrm{U}$ \\
\hline$[41]$ & $\mathrm{S}$ & & & $\mathrm{D}$ \\
\hline$[42]$ & $\mathrm{S}$ & & & $\mathrm{D}$ \\
\hline$[43]$ & S & & & $\mathrm{U}$ \\
\hline$[44]$ & $\mathrm{S}$ & D & & $\mathrm{U}$ \\
\hline
\end{tabular}




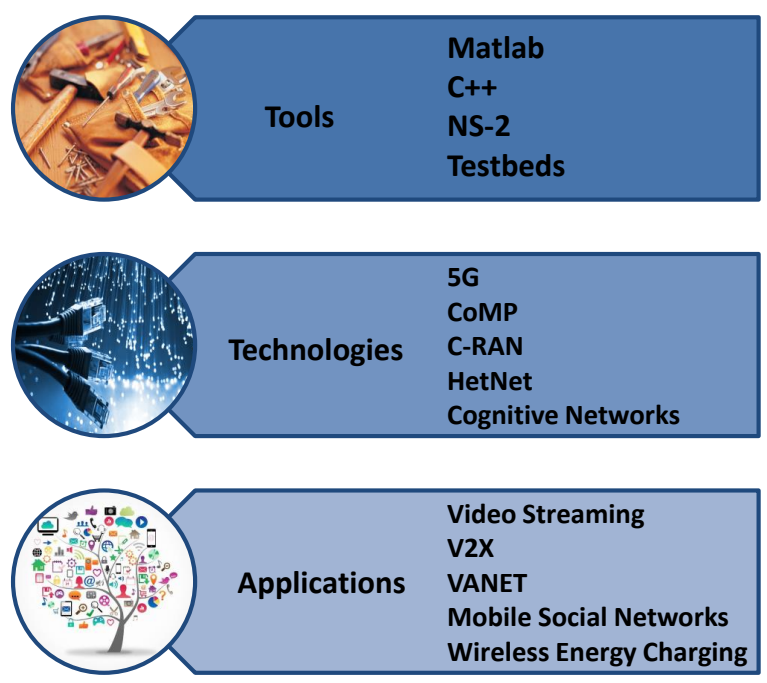

Figure 3.3: Tools, technologies and applications of D2D resource allocation.

hub for D2D communications and therefore, its function requires the knowledge of CSI to facilitate smooth transition between different communications. Our objective is a singular one, solely focused on the maximization of sum throughput.

\subsection{Tools, Technologies and Applications of D2D Resource Allocation}

In this section, we will analyze the tools and technologies used in the literature and some of the applications for D2D resource allocation. We have already briefly touched the topic of D2D applications in Chapter 2; however, the focus was merely on the application layer while in this section we will discuss about the application of D2D resource allocation in the physical layer, especially when incorporated into different networks. Fig. 3.3 gives a summary of the information we have extracted from the papers in the literature. In the following, we will discuss about each topic respectively. 
A. Tools: For simulation tools, most researchers chose MATLAB to solve the optimization problem of resource allocation in D2D communications as it is a universal tool for model building, numerical simulation and implementation. However, several papers implemented their proposed wrok in $\mathrm{C}++[36]$ or in NS-2, a network simulation platform which recently has gained a lot of momentum in the academia. Few have designed their own testbeds for implementation.

B. Technologies: The technologies used in the literature are kaleidoscopic as well, consisting of cognitive radio, HetNets, C-RAN, etc. It further proves the interconnection among various technologies and techniques. Many papers set up their system model in LTE/LTE-A [9,10,24,25,39, 41,44] while few built their models for 5G networks [37]. Full duplex D2D communications scenarios were addressed in [11,38]. In [31], a cognitive radio based joint resource allocation and power control scheme for D2D communications is offered to assist multicast services. In [6], a resource allocation approach for D2D communications in C-RAN based LTE-A networks was proposed that exploits the hybrid structure of C-RAN networks to achieve energy efficiency while guaranteeing QoS. In [5,32], resource allocation for D2D in heterogeneous networks (HetNets) was discussed. Authors in [32] devised a dynamic programming approach to allocate upload and download traffic efficiently in an interference-aware manner thanks to D2D support. In [5], the authors combined mode selection and power control in a HetNet comprised of both cellular and D2D transmission modes and form a fractional non-convex problem. This approach takes into consideration multiple constraints and proves to be mathematically efficient. All of the technologies mentioned above, like D2D communications, will play a larger role in $5 \mathrm{G}$ communications than they already have and it is a good sign that they not only can facilitate the development of D2D communications but also benefit from the assistance of D2D communications.

C. Applications: The applications of D2D resource allocation discussed in the collected literature run the gamut from video streaming [28] to wireless energy charging. Applications of D2D communications in vehicle-to-everything (V2X) communications [15], VANETs [25] and mobile social networks (MSNs) [43] were also investigated to assist in satisfying QoS. In [15], the authors provided a solution to radio resource management in a D2D-based V2X application by analyzing and transforming the V2X latency and reliability requirements into a resource allocation problem. In [25], a similar attempt to facilitate VANETs through D2D communications was discussed, where the authors divided the vehicles on the road into multiple clusters. In [43], the authors proposed an application of MSNs by setting up D2D mode for offline users. Then they applied game theory 
to achieve efficient data transmission among local users. As the development of novel technologies and techniques, we are poised to witness an explosion of D2D related applications in the future.

\subsection{Energy Harvesting and Resource Allocation in Underlay D2D Communications}

Energy is everywhere. From every step we make on the street, to the sunshine and breeze we feel on our face, we can feel energy around us almost every second. However, not all energy can be utilized efficiently. In fact, nature has offered us tremendous amount of energy resources rarely tapped by human beings. Instead, we squandered tons of fossil fuel, which has turned out to be the main contributor to global warming. To save energy for earth and cost for business, it is imperative for us to fully exploit many under-utilized or unused energy sources. This is where energy harvesting comes in. Energy harvesting, by its definition, refers to the process by which energy, mostly from ambient sources (solar energy, thermal energy, kinetic energy, etc), is captured and stored in small, wireless devices for future use.

Wireless energy harvesting technology is already well established in the building of automation sector and is on its way to power many smart applications. In wireless sensor networks (WSN), people have incorporated dedicated wireless transmitters into the system to tackle the issue of limited battery lives. Like WSN, the performance of D2D communications can be heavily affected by battery lives as well since these delicate devices will simply stop working once their batteries are drained. Add to it the fact that replacing the tiny batteries of these devices takes more time and human resources than we would rather spend, the pioneering idea to prolong the lifetime of the system by incorporating energy harvesting into the system is not that surprising. Several papers in the academia have started exploring this topic, which can be found in [45-52].

In [45], Ahmed et al. utilize tools from stochastic geometry to model and analyze energyharvesting-based D2D communications in cellular networks. In the model, D2D transmitters harvest energy from ambient interference and then reuse channels of CUs to communicate with their corresponding receivers. Two spectrum access policies - random spectrum access (RSA) policy and prioritized spectrum access (PSA) policy - are employed for communications in the uplink or downlink respectively, where different network outage probabilities are given to show that energy harvesting can be a reliable alternative source to underlay D2D communications. 
Following similar theories of stochastic geometry, a model of D2D communications powered by $\mathrm{EH}$ in heterogeneous cellular network (D2D-EHHN) is proposed by Howard et al. in [46]. They then introduce the concept of EH region (EHR) to develop a framework to analyze D2D-EHHN. Finally, the network outage probability is derived in close form to measure the system performance. Simulation results show that higher EH efficiency is more likely to enhance the system performance, though the improvement is not guaranteed.

In [47], Zhenyu et al. attempt to optimize the energy efficiency of a D2D system with dynamic and sporadic energy arrivals. They model the optimization problem as a non-cooperative game over a finite horizon. Then an iterative power allocation algorithm of relatively low complexity is proposed with the aid of nonlinear fractional programming and Lagrange dual decomposition. Numeric results show that the proposed algorithm is better than a greedy algorithm.

Table 3.3: Energy harvesting in D2D communications

\begin{tabular}{|c|c|c|c|c|c|}
\hline & $\begin{array}{l}\text { Underlay / } \\
\text { Overlay }\end{array}$ & $\begin{array}{l}\text { Uplink / } \\
\text { Downlink }\end{array}$ & $\begin{array}{c}\text { Causal / } \\
\text { Non-causal }\end{array}$ & Objective & Algorithm \\
\hline Ref. & $\mathrm{U} / \mathrm{O}$ & $\mathrm{U} / \mathrm{D}$ & $\mathrm{C} / \mathrm{N}$ & & \\
\hline$[45]$ & $\mathrm{U}$ & $\mathrm{U} \& \mathrm{D}$ & $\mathrm{N}$ & $\mathrm{N} / \mathrm{A}$ & $\begin{array}{l}\text { Statistic approach } \\
\text { based on stochastic } \\
\text { geometry }\end{array}$ \\
\hline$[47]$ & $\mathrm{U}$ & U\&D & $\mathrm{N}$ & $\begin{array}{l}\text { Maximize energy } \\
\text { efficiency }\end{array}$ & $\begin{array}{l}\text { Lagrangrian dual } \\
\text { decomposition }\end{array}$ \\
\hline$[48]$ & $\mathrm{U}$ & $\mathrm{U}$ & $\mathrm{N}$ & $\begin{array}{l}\text { Minimize sum } \\
\text { transmit power }\end{array}$ & $\begin{array}{l}\text { Lagrangian } \\
\text { multiplier method }\end{array}$ \\
\hline$[49]$ & $\mathrm{U}$ & D & $\mathrm{C} \& \mathrm{~N}$ & $\begin{array}{l}\text { Maximize sum } \\
\text { throughput }\end{array}$ & $\begin{array}{l}\text { Lagrangian } \\
\text { multiplier method }\end{array}$ \\
\hline$[50]$ & $\mathrm{O}$ & $\mathrm{D}$ & $\mathrm{N}$ & $\begin{array}{l}\text { Maximize sum } \\
\text { throughput }\end{array}$ & $\begin{array}{l}\text { Complex } \\
\text { optimization tool }\end{array}$ \\
\hline$[52]$ & $\mathrm{U}$ & U\&D & $\mathrm{N}$ & $\mathrm{N} / \mathrm{A}$ & $\begin{array}{l}\text { Statistic approach } \\
\text { based on stochastic } \\
\text { geometry }\end{array}$ \\
\hline
\end{tabular}

In [50], solar energy harvesting is exploited in the D2D system. Umber et al. formulate a 
sum rate maximization problem for solar energy harvesting based overlay in-band D2D network. Energy and power constraints are considered. The analysis is based on the real time solar EH data. The proposed problem is NP-hard. To solve it, the authors utilize the complex optimization tool and analyze the model with real solar energy harvesting data. Results further corroborate that both power allocation and energy arrival scheduling are effective in yielding higher throughput performance.

Table 3.3 summarizes the characteristics, objectives of problems and algorithms used to solve the problems in energy harvesting in D2D communications. Two common energy harvesting models are stochastic models and deterministic models [53]. In deterministic models, full knowledge of energy arrival instants and amounts is known in advance by transmitters whereas in scholastic models, energy refill is considered as random processes. We also use the terms like causal and non-causal to refer to the nature of the energy harvesting process. From the table, we can see that most papers related to energy harvesting in D2D communications consider non-causal energy harvesting from ambient source in their system models where energy arrivals are intermittent and considered as a stochastic process and the amount of energy harvested every time is not assured. Causal model is only considered in the online joint optimization algorithm in [49]. Our paper considers a deterministic [54] energy harvesting model in D2D communications. A dedicated, omni-directional wireless power transmitter is located at the base station for the sake of simplicity. It could be established anywhere inside the cell for charging the batteries of wireless sensors as shown in [55], but this paper does not deal with optimal position of wireless power transmitters.

\subsection{Summary}

In this chapter, we provided a thorough survey of resource allocation schemes in underlay D2D communications. After the review of dozens of state-of-the-art papers related to such topic, we managed to analyze from different angles the issues of D2D resource allocation through charts and tables. Our discussion included the objectives and constraints of D2D resource allocation problems, the problem types and potential algorithms to solve these problems, the system characteristics of these D2D models, and finally an intensive discussion of resource allocation in energy harvesting incorporated underlay D2D systems.

In the next chapter, we will present our own energy harvesting aided D2D system model. We 
will also formulate an optimization problem based on mode selection, resource block allocation and power allocation. Then we will provide a deep analysis of the formulated problem and adopt the NOMAD algorithm from the OPTI tool box to solve it. Preliminary results will be presented to show the superiority of our system and algorithm. 


\section{Chapter 4}

\section{Energy Harvesting Assisted D2D Communcations}

In this chapter, we present an energy harvesting assisted D2D communications system. First, we construct our system model based on the assumptions and restrictions we learned from the similar papers of this topic in the literature. Then we formulate an optimization problem whose goal is to maximize sum throughput of D2D users over a certain period of time under such complex system. We consider typical constraints in terms of transmit power for D2D users, QoS requirement for cellular users, resource block sharing rules, etc. Since our system is centralized, we cannot provide an optimal solution for an arbitrary number of time slots. Therefore, we focus on producing a sub-optimal solution, which amounts the summation of optimal solution during each time slot. The problem is a mixed integer non-linear programming (MINLP) problem. We provide an optimal solution using NOMAD (Nonlinear Optimization by Mesh Adaptive Direct Search) algorithm inside the OPTI toolbox in MATLAB. The algorithm can help us solve both the resource block allocation and power allocation altogether. We then offer a thorough analysis of the preliminary results in terms of percentage of active D2D users, sum throughput of D2D users under various simulation parameters without jeopardizing the QoS requirements of cellular users and violating the transmit power limitation of D2D users. 


\subsection{System Model and Problem Formation}

In this section, we present in detail the system model we used in the formation of our optimization problem, whose objective is to maximize sum throughput of D2D users underlaying cellular communications based on energy harvesting.

\subsubsection{System Model}

We consider the existence of two types of wireless communications, cellular communications and D2D communications, in the model. The assumption is that each cellular user (CU) occupies a dedicated resource block (RB) and works as a primary user while D2D users reuse the RBs of CUs to work as secondary users. We suppose there are $M$ pairs of D2D users with the $i_{t h}$ D2D pair denoted as $D 2 D_{i}$ and $N$ cellular users with the $j_{t h}$ cellular user represented as $C U_{j}$. Fig. 4.1 demonstrates a simple scenario composed of $M=4$ D2D pairs and $N=4$ CUs. To simplify the model, only downlink scenarios are considered in the procedure of data transmission and energy harvesting. From the figure, we can see the transmission links and interference links. We can also see that D2D links are powered by energy harvested from the wireless power transmitter located at the BS and reuse the RBs of ongoing cellular transmission links.

In our system, the cellular communications is enabled all the time, but D2D communications is only available when the D2D pair has enough energy to support the data transmission. To facilitate the description of our model, we divide the operating time of D2D users into $K$ time slots of equal duration with the $k_{t h}$ time slot signified as $\tau_{k}$. We also introduce $E_{i, k}$ to represent the energy level for $D 2 D_{i}$ after $\tau_{k} . E_{i, 0}$ as a constant signifies the initial energy level for $D 2 D_{i}$ before transmissions in any time slot. The update function of two states - data transmitting state and energy harvesting state - for D2D pairs is shown in Fig. 4.2. $E_{C o n}(i, k)$ and $E_{H a r}(i, k)$ refer to the energy harvested or consumed by $D 2 D_{i}$ in $\tau_{k}$ respectively. Their mathematical expressions are given below:

$$
\begin{aligned}
& E_{C o n}(i, k)=P_{i, k} * \tau_{k}, \\
& E_{H a r}(i, k)=\kappa P_{H} *\left(d_{i, B}\right)^{-\alpha} * \tau_{k} * \eta,
\end{aligned}
$$

where $P_{i, k}$ denotes the transmit power of $D 2 D_{i}$ during $\tau_{k}, P_{H}$ denotes the transmit power from wireless power transmitter located at the BS for harvesting; $d_{i, B}$ represents the distance between a $D 2 D_{i}$ and the BS, $\kappa$ and $\alpha$ represent the path loss constant and path loss exponent respectively 


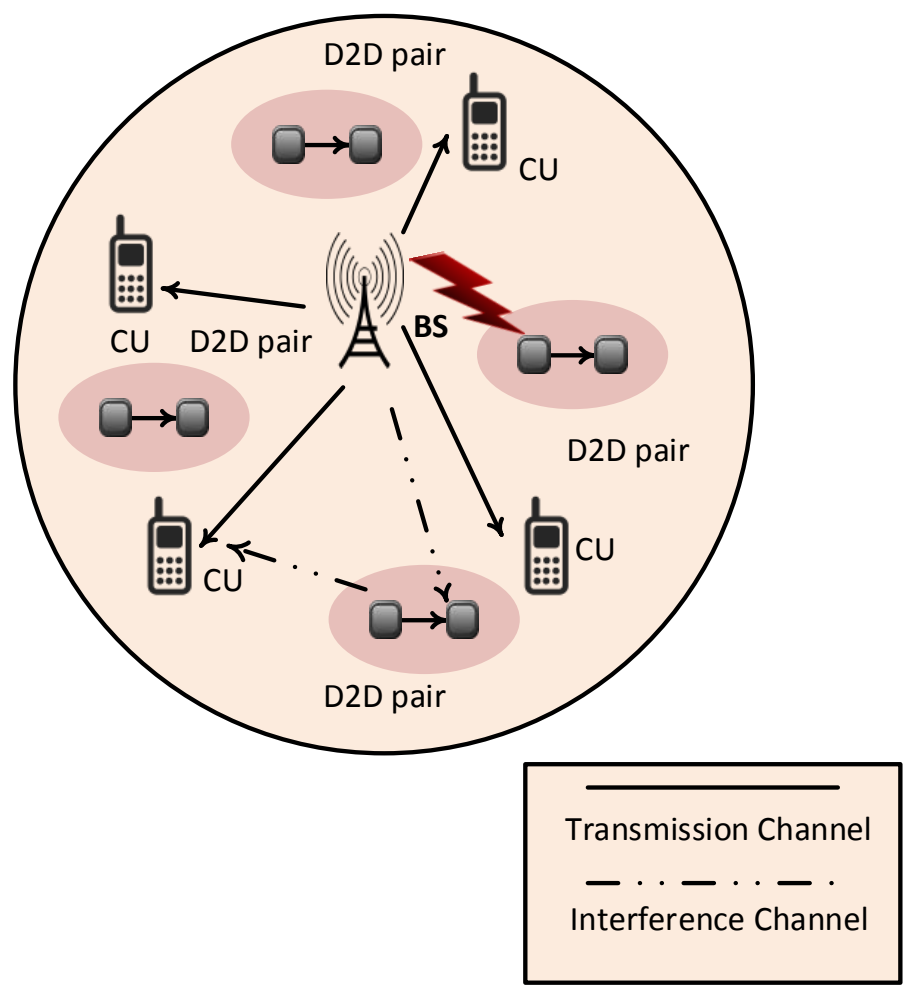

Figure 4.1: A simple scenario of system model.

and $\eta$ represents the energy harvesting efficiency factor. Note that $\mathrm{D} 2 \mathrm{D}$ pairs will not impose interference on CUs during EH mode since we assume there exist dedicated RBs for D2D energy harvesting.

At the beginning of every time slot, all D2D pairs will update their energy level to the BS. The BS will then compare the values with two threshold values to make a decision about their states and and wait for their acknowledgement message before any real action takes place. If the remaining energy of a D2D pair goes below a lower threshold $E_{\text {low }}$, the BS will send a 'HARV' to it and initiate energy harvesting process once it receives the 'HACK' message. Similarly, if the remaining energy of a D2D pair bounces back to an upper threshold $E_{u p}$ after some time slots of harevsting, it will toggle back into data transmitting state from energy harvesting state. BS will send out 'TRSx' message and wait for the acknowledgement message 'TRACK'. Here ' $x$ ' is a number that represents 


$$
E_{i, k+1}=E_{i, k}+E_{\text {Har }}(i, k)
$$

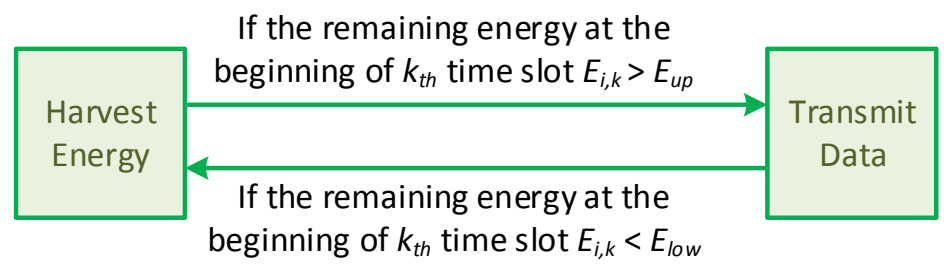

$$
E_{i, k+1}=E_{i, k}-E_{\text {con }}(i, k)
$$

Figure 4.2: A diagram of two states for D2D pairs.

the RB that the said D2D pair will reuse. Note that the BS will first put all available D2D pairs in a list of active users and allocate the RBs to them altogether before sending out the decision. The time needed to make the decision and exchange information on whether to transmit or harvest can be neglected as it is very small compared with the whole time slot. The whole process is delineated in Fig. 4.3.

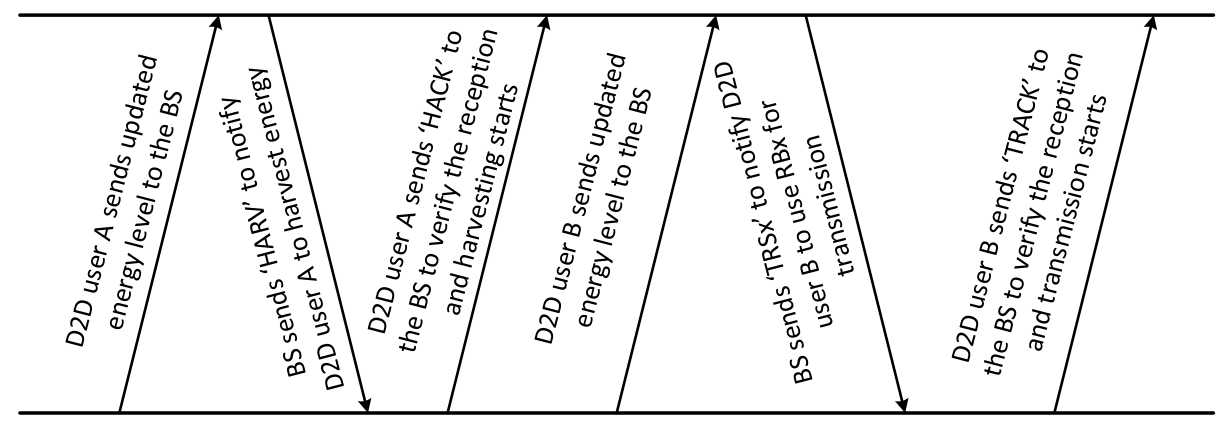

Figure 4.3: Message exchange for mode selection. 
Therefore, for a D2D pair $D 2 D_{i}$, we can use the expression below to show which mode it is in during time slot $\tau_{k}$ :

$$
\gamma_{i, k}= \begin{cases}1, & \text { if } D 2 D_{i} \text { transmits data during } \tau_{k} \\ 0, & \text { if } D 2 D_{i} \text { harvests energy during } \tau_{k} .\end{cases}
$$

In each time slot, the relationship between D2D pairs and cellular users in terms of RB sharing is expressed as:

$$
\phi_{i j, k}= \begin{cases}1, & \text { if } D 2 D_{i} \text { shares } C U_{j} \text { 's RB during } \tau_{k} \\ 0, & \text { otherwise. }\end{cases}
$$

Note that here $\phi_{i j, k}$ as defined above represents the realistic relationship between D2D pairs and cellular users. The value of $\phi_{i j, k}$ may vary as $k$ varies since the number of active D2D pairs will experience an update at the beginning of every time slot. Due to the limitation of interference, we assume in our model one RB of every CU can only be shared by one D2D pair in every time slot if any, which can be expressed as:

$$
\sum_{i=1}^{M} \phi_{i j, k} \leqslant 1 \quad \forall j \in N, k \in K .
$$

We assume interference channels to be AWGN channels. Then we define $h_{i j}$ and $h_{j}$ as the transmission channel gains between $D 2 D_{i}$ over the $\mathrm{RB}$ of $C U_{j}$ and the channel gains between $\mathrm{BS}$ and $C U_{j}$. Similarly, we can define $h_{j i}^{I}$ and $h_{i j}^{I}$ as the interference channel gains of BS and $C U_{j}$ at the $D 2 D_{i}$ receiver and the interference channel gains from the $D 2 D_{i}$ transmitter to the $j_{t h} \mathrm{CU}$. The expression for the data rate of $D 2 D_{i}$ over the $\mathrm{RB}$ of $C U_{j}$ in $\tau_{k}$ can be formulated as:

$$
r_{i j, k}=W \log _{2}\left(1+\frac{P_{i j, k} h_{i j}}{N_{0}+P_{j} h_{j i}^{I}}\right),
$$

where $P_{i j, k}$ represents the transmit power of $D 2 D_{i}$ over the RB of $C U_{j}$ during $\tau_{k}$ and $P_{j}$ represents the transmit power of $C U_{j}$. Therefore, the sum data rate of $D 2 D_{i}$ during the $\tau_{k}$ time slot can be further expressed as:

$$
r_{i, k}=\sum_{j=1}^{N} \phi_{i j, k} r_{i j, k}=W \sum_{j=1}^{N} \phi_{i j, k} \log _{2}\left(1+\frac{P_{i j, k} h_{i j}}{N_{0}+P_{j} h_{j i}^{I}}\right) .
$$

Since D2D users are secondary users, we also need to lower the interference D2D users imposed on CUs so that it will not affect the QoS performance of CUs severely. The interference of the $C U_{j}$ 
suffered from the D2D user with whom it shares the RB during $\tau_{k}$ is represented as:

$$
I_{j, k}=\sum_{i=1}^{M} \phi_{i j, k} P_{i j, k} h_{i j}^{I} .
$$

Following this, we give out a constraint of QoS requirement for CUs shown below. The data rate of any cellular user should be greater than or equal to the threshold value $R_{C}$ as expressed below:

$$
W \log _{2}\left(1+\frac{P_{j} h_{j}}{N_{0}+I_{j, k}}\right) \geqslant R_{C} \quad \forall j \in N, k \in K
$$

\subsubsection{Problem Formation}

Based on the system model and assumptions mentioned above, the problem we formulate is presented as next, the goal of which is to maximize the sum throughput of $M$ D2D pairs over $K$ time slots in regard to four optimization elements: $\gamma_{i, k}, \phi_{i j, k}, P_{i j, k}$ and $P_{j}$.

$$
\begin{gathered}
\max \sum_{k=1}^{K} \sum_{i=1}^{M} \gamma_{i, k} r_{i, k}=\Omega\left(\gamma_{i, k}, \phi_{i j, k}, P_{i j, k}, P_{j}\right) \\
\text { s.t. } \sum_{i=1}^{M} \phi_{i j, k} \leqslant 1 \quad \forall j \in N, k \in K \\
W \log _{2}\left(1+\frac{P_{j} h_{j}}{N_{0}+I_{j, k}}\right) \geqslant R_{C} \quad \forall j \in N, k \in K \\
0 \leqslant P_{i, k}=\sum_{j=1}^{N} P_{i j, k} \leqslant P_{\max } \quad \forall i \in M, k \in K, \\
\gamma_{i, k} \in\{0,1\}, \phi_{i j, k} \in\{0,1\} \quad \forall i \in M, j \in N, k \in K,
\end{gathered}
$$

where (4.10a) signifies the limitation that one RB can be allocated to only one D2D pair, (4.10b) is the data rate requirement for cellular users, and (4.10c) means that the transmit power for $D 2 D_{i}$ over $\tau_{k}$ cannot exceed the maximal transmit power of D2D users $P_{\max }$. represents the obvious binary integer restriction for $\gamma_{i, k}$ and $\phi_{i j, k}$.

The value of $K$ is yet defined. However, we can briefly discuss the effect of the chosen $K$ on the whole problem. Apparently, if $K$ is too small, then we would not have enough time to drain the energy to the level below $E_{l o w}$ and therefore the whole energy harvesting process would not be initiated. Therefore, we need to choose the value of $K$ large to run through a full cycle of energy 
harvesting and data transmission at least once. To get this value, we would need to do some simple test before settling down in our simulation section.

We can simply start by calculating the average energy consumption per time slot for D2D users using random resource allocation technique. Since with random resource allocation the power efficiency will not be higher than the optimal resource allocation we are trying to get, the average energy consumption per time slot we get will not be lower than ideal power consumption. We can rely on this value to calculate how many time slots it will probably take to initiate the energy harvesting process for the first time. Similarly, we can obtain how many time slots it will usually take to harvest enough energy so that the D2D user will go back to data transmission mode.

Since the value of $K$ is arbitrary, there is no way in real-time system the BS can optimize over this value due to unpredictability and complexity. Therefore, we divide the optimization problem into $K$ instances by rewriting the objective as shown below so that the BS can solve each of them individually while at the same get rid of one optimization element $\gamma_{i, k}$. These $K$ instances are connected to each other because the energy left from one time slot $E_{i, k}$ will be used to determine the mode of the next time slot $E_{i, k+1}$ at the beginning of next time slot.

$$
\begin{aligned}
& \max \sum_{k=1}^{K} \sum_{i=1}^{M} \gamma_{i, k} r_{i, k} \\
= & \max \sum_{k=1}^{K} \gamma_{i, k} \sum_{i=1}^{M} r_{i, k} \\
= & \sum_{k=1}^{K} \gamma_{i, k} \max \sum_{i=1}^{M} r_{i, k} \\
= & \sum_{k=1}^{K} \gamma_{i, k} \max \sum_{i=1}^{M} \sum_{j=1}^{N} \phi_{i j, k} r_{i j, k}
\end{aligned}
$$

After this, we can focus on solving each instance of the resource allocation problem individually. As discussed, each problem is matched with each time slot. We can then update the battery energy level based on the allocation within the last time slot. To simply the notation in discussion, we use a general $k^{*}$ to refer a random time slot. 
Table 4.1: Description of the symbols used in the model.

\begin{tabular}{ll}
\hline Symbol & Description \\
\hline$M$ & Number of D2D pairs \\
$N$ & Number of CUs \\
$K$ & Number of time slots \\
$D 2 D_{i}$ & The $i_{t h}$ D2D pair \\
$C U_{j}$ & The $j_{t h}$ CU \\
$\tau_{k}$ & The $k_{t h}$ time slot \\
$\gamma_{i, k}$ & Parameter that represents whether the mode of $D 2 D_{i}$ over $\tau_{k}$ \\
$\phi_{i j, k}$ & Parameter that denotes whether $D 2 D_{i}$ is using the RB of $C U_{j}$ over $\tau_{k}$ \\
$P_{j}$ & Transmit power of $C U_{j}$ \\
$P_{i j, k}$ & Transmit power of $D 2 D_{i}$ using the RB of $C U_{j}$ over $\tau_{k}$ \\
$P_{i, k}$ & Total transmit power of $D 2 D_{i}$ over $\tau_{k}$ \\
$P_{H}$ & Omni-directional transmit power for energy harvesting from the central source \\
$P_{\text {max }}$ & Maximal transmit power for each D2D user \\
$h_{i j}$ & Channel gains between $D 2 D_{i}$ over the RB of $C U_{j}$ \\
$h_{j}$ & Channel gains between BS and $C U_{j}$ \\
$h_{j i}^{I}$ & Interference channel gains from BS to the $i_{t h}$ D2D receiver over the RB of $C U_{j}$ \\
$h_{i j}^{I}$ & Interference channel gains from the $i_{t h}$ D2D transmitter to $C U_{j}$ \\
$r_{i j, k}$ & Data rate of $D 2 D_{i}$ over the RB of $C U_{j}$ in $\tau_{k}$ \\
$E_{i, k}$ & Energy level of $D 2 D_{i}$ after $\tau_{k}$ \\
$E_{u p}$ & Upper energy threshold for D2D users \\
$E_{m a x}$ & Maximal energy for D2D batteries \\
$E_{l o w}$ & Lower energy threshold for D2D users \\
$R_{C}$ & Energy harvesting efficiency factor \\
$\eta$ & \\
\hline &
\end{tabular}

Therefore, the original problem has now be successfully divided into $K$ instances with its most generic form represented as: 


$$
\begin{gathered}
\max \sum_{i=1}^{M} \sum_{j=1}^{N} \phi_{i j, k^{*}} r_{i j, k^{*}}=\Omega\left(\phi_{i j, k^{*}}, P_{i j, k^{*}}, P_{j}\right) \\
\text { s.t. : } \sum_{i=1}^{M} \phi_{i j, k^{*}} \leqslant 1 \quad \forall j \in N \\
W \log _{2}\left(1+\frac{P_{j} h_{j}}{N_{0}+I_{j, k^{*}}}\right) \geqslant R_{C} \quad \forall j \in N, \\
0 \leqslant P_{i, k^{*}}=\sum_{j=1}^{N} P_{i j, k^{*}} \leqslant P_{\max } \quad \forall i \in M, \\
\phi_{i j, k^{*}} \in\{0,1\} \quad \forall i \in M, j \in N,
\end{gathered}
$$

where (4.13b) refers to the QoS requirement for cellular users in a generic time slot $\tau_{k^{*}}$, (4.13c) refers to the transmit power constraint for D2D users in a generic time slot $\tau_{k^{*}}$, and (4.13d) is the restriction imposed by the $\mathrm{RB}$ sharing rule.

For constraint (4.13b), we can get rid of the logarithmic expression by dividing both sides by $W$ and then calculating the exponential of 2 . We can eventually simplify the inequality as below:

$$
P_{j} \geqslant \frac{\left(2^{\frac{R_{C}}{W}}-1\right)\left(N_{0}+I_{j, k^{*}}\right)}{h_{j}} \quad \forall j \in N
$$

In order to have maximal sum throughput for D2D users, apparently the smaller the transmit power for cellular users, the easier it is to achieve such an optimal value, because smaller transmit power for cellular users will mitigate the interference cellular communications will exert on the active D2D links. Therefore, when $P_{j}$ is equal to the expression on the right in (4.14), we get the optimal value for $P_{j}$ signified as $P_{j}^{*}$.

For next step, we can bring $P_{j}^{*}$ back into the objective (4.11d) to get rid of the optimization element $P_{j}$. We get: 


$$
\begin{aligned}
& \max \sum_{i=1}^{M} \sum_{j=1}^{N} \phi_{i j, k^{*}} r_{i j, k^{*}} \\
= & \max W \sum_{i=1}^{M} \sum_{j=1}^{N} \phi_{i j, k^{*}} \log _{2}\left(1+\frac{P_{i j, k^{*}} h_{i j}}{N_{0}+P_{j} h_{j i}^{I}}\right) \\
= & \max W \sum_{i=1}^{M} \sum_{j=1}^{N} \phi_{i j, k^{*}} \log _{2}\left(1+\frac{P_{i j, k^{*}} h_{i j}}{N_{0}+\frac{\left(2 \frac{R_{C}}{W}-1\right)\left(N_{0}+I_{j, k^{*}}\right) h_{j i}^{I}}{h_{j}}}\right) \\
= & \max W \sum_{i=1}^{M} \sum_{j=1}^{N} \phi_{i j, k^{*}} \log _{2}\left(1+\frac{A_{i j} P_{i j, k^{*}}}{B_{i j}+\sum_{i=1}^{M} C_{i j} \phi_{i j, k^{*}} P_{i j, k^{*}}}\right) \\
= & \Omega\left(\phi_{i j, k^{*}}, P_{i j, k^{*}}\right)
\end{aligned}
$$

where $A_{i j}=h_{i j} h_{j}, B_{i j}=\left[\left(2^{\frac{R_{C}}{W}}-1\right) h_{j i}^{I}+h_{j}\right] * N_{0}$ and $C_{i j}=\left(2^{\frac{R_{C}}{W}}-1\right) h_{j i}^{I} h_{i j}^{I}$.

Since the RB of one cellular user can only be reused by one D2D link, as demonstrated by (4.10a), so if $\phi_{i j, k^{*}}=1$ is true for one particular D2D pair $D 2 D_{i^{*}}$, then $\phi_{i j, k^{*}}=0$ will be true for all D2D pairs except $D 2 D_{i^{*}}$. Therefore, we can simplify the $\sum_{i=1}^{M} C_{i j} \phi_{i j, k^{*}} P_{i j, k^{*}}$ in (4.15d) to $C_{i j} \phi_{i j, k^{*}} P_{i j, k^{*}}$ as the rest components in the summation are equal to zero in this case and thus redundant.

So now the objective function has been simplified into one with only two resource allocation elements: $\phi_{i j, k^{*}}$ and $P_{i j, k^{*}}$, which corresponds to the allocation of RBs and power respectively. However, the problem is still a mixed integer nonlinear programming (MINLP) problem, and it remains mathematically strenuous to solve it directly. For example, in terms of RB allocation, we have $M$ D2D users and $N$ cellular RBs $(M \leqslant N)$. In order to make sure that each D2D pair has at least one RB to function, we will have $M^{N}-\sum_{X=1}^{M-1} C_{M}^{X} X^{N}$ possible solutions. This is unsolvable by brute-force since the complexity can be scaled very easily based on the choice of $M$ and $N$. Therefore, the best way to solve this problem is to employ some established algorithms specifically suited for MINLP problems. In the next section, we will introduce the OPTI toolbox in MATLAB and one particular algorithm called NOMAD (Nonlinear Optimization by Mesh Adaptive Direct Search), which we choose to use in solving this complicated problem. 


\subsection{OPTI Toolbox and NOMAD Algorithm}

OPTimization Interface (OPTI) Toolbox is a free MATLAB toolbox for constructing and solving linear, nonlinear, continuous and discrete optimization problems. A range of open source and academic solvers are supplied with no compilation required. In this case, we choose the NOMAD solver to solve the MINLP problem. NOMAD means nonlinear optimization with MADS (Mesh Adaptive Direct Search) algorithm. It is commonly used to solve non-differentiable and global nonlinear programs as well as non-convex MINLPs, and does this quite well for problems up to a few hundred variables. NOMAD is at its best when applied to black box functions. Such functions are typically the result of expensive computer simulations which have no exploitable property such as derivatives and may be contaminated by noise or fail to give a result even for feasible points.

At the core of NOMAD resides the Mesh Adaptive Direct Search (MADS) algorithm. As the name implies, this method generates iterates on a series of meshes with varying size. A mesh is a discretization of the space of variables. However, also as the name implies, the algorithm performs an adaptive search on the meshes including controlling the refinement of the meshes. The objective of each iteration of the MADS algorithm, is to generate a trial point on the mesh that improves the current best solution. When an iteration fails to achieve this, the next iteration is initiated on a finer mesh. Each iteration is composed of two principal steps called the search and the poll steps. The search step is crucial in practice because it is so flexible, but it is a difficulty for the theory for the same reason. The search can return any point on the underlying mesh, but of course, it is trying to identify a point that improves the current best solution. The poll step is more rigidly defined, though there is still some flexibility in how this is implemented. The poll step generates trial mesh points in the vicinity of the best current solution. Since the poll step is the basis of the convergence analysis, it is the part of the algorithm where most research has been concentrated.

So how did the simulation process actually work? In order to run the NOMAD solver, we have to construct an optimization function initially based on the required format of input variables for OPTI toolbox. Suppose we have $M$ D2D pairs and $N$ cellular users, we will end up with $M * N * 2$ variables in the optimization process, with $M * N$ consecutive variables representing power allocation $P_{i j, k^{*}}$ and $M * N$ binary variables for RB selection $\phi_{i j, k^{*}}$. We also need to construct $M * N * 2$ inequality constraints for these variables individually following the requirements imposed in (4.13c) and (4.13d). The pseudo code for the entire NOMAD simulation is shown in Algorithm 1 and 2. 
Algorithm 1 is the main program for energy harvesting assisted D2D communications and algorithm 2 is the actual program of NOMAD optimization, which we would need to call numerous times in our entire simulation. As we can observe clearly, at the beginning of every time slot $\tau_{k}$, we will check the energy level in all D2D pairs and put the ones that are able for data transmission in a list called AcList(k). For all D2D pairs in the list, we execute joint power and resource allocation based on NOMAD, which will return us the optimal resource allocation combination. We then we update the sum throughput and energy levels for all D2D users.

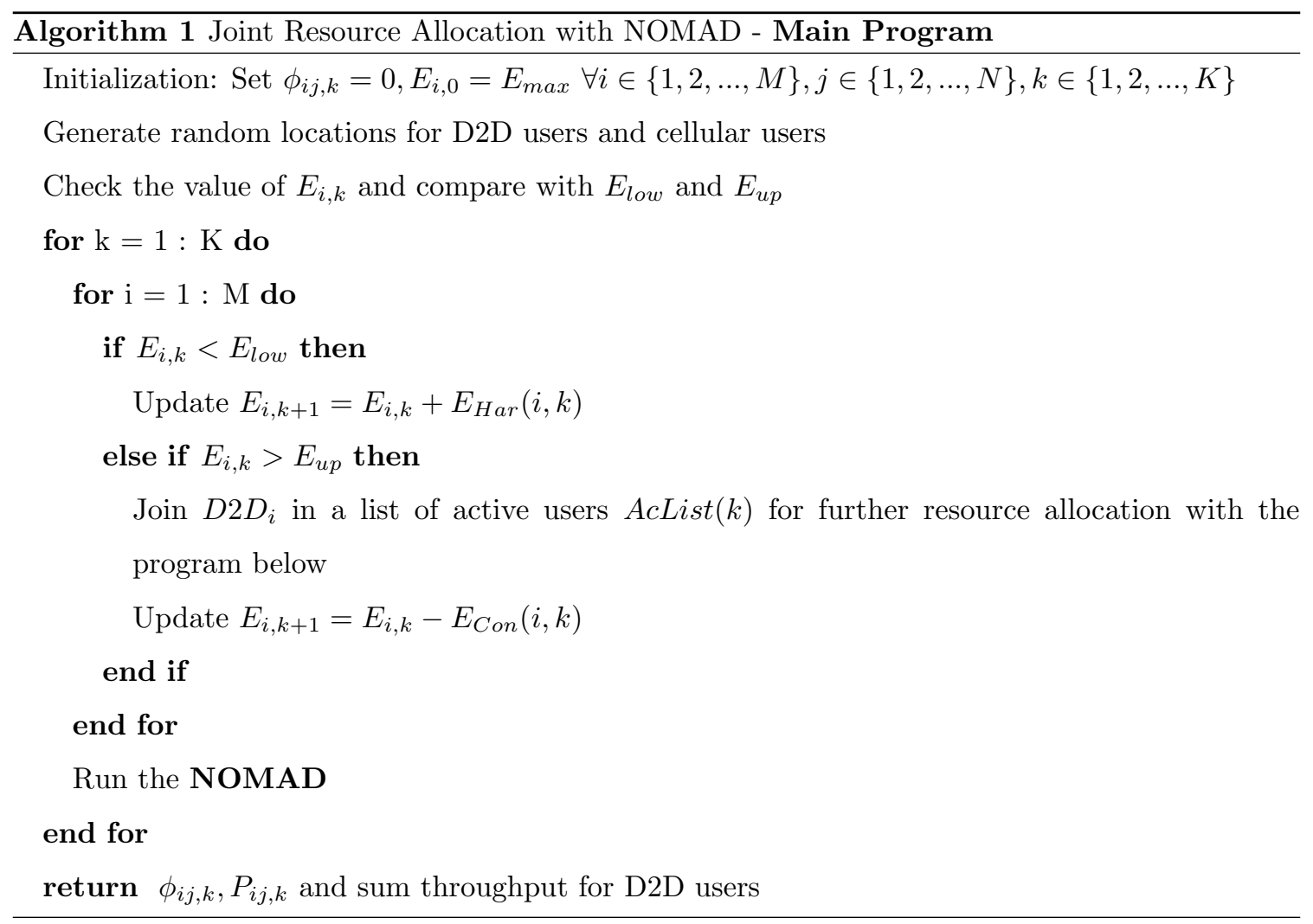




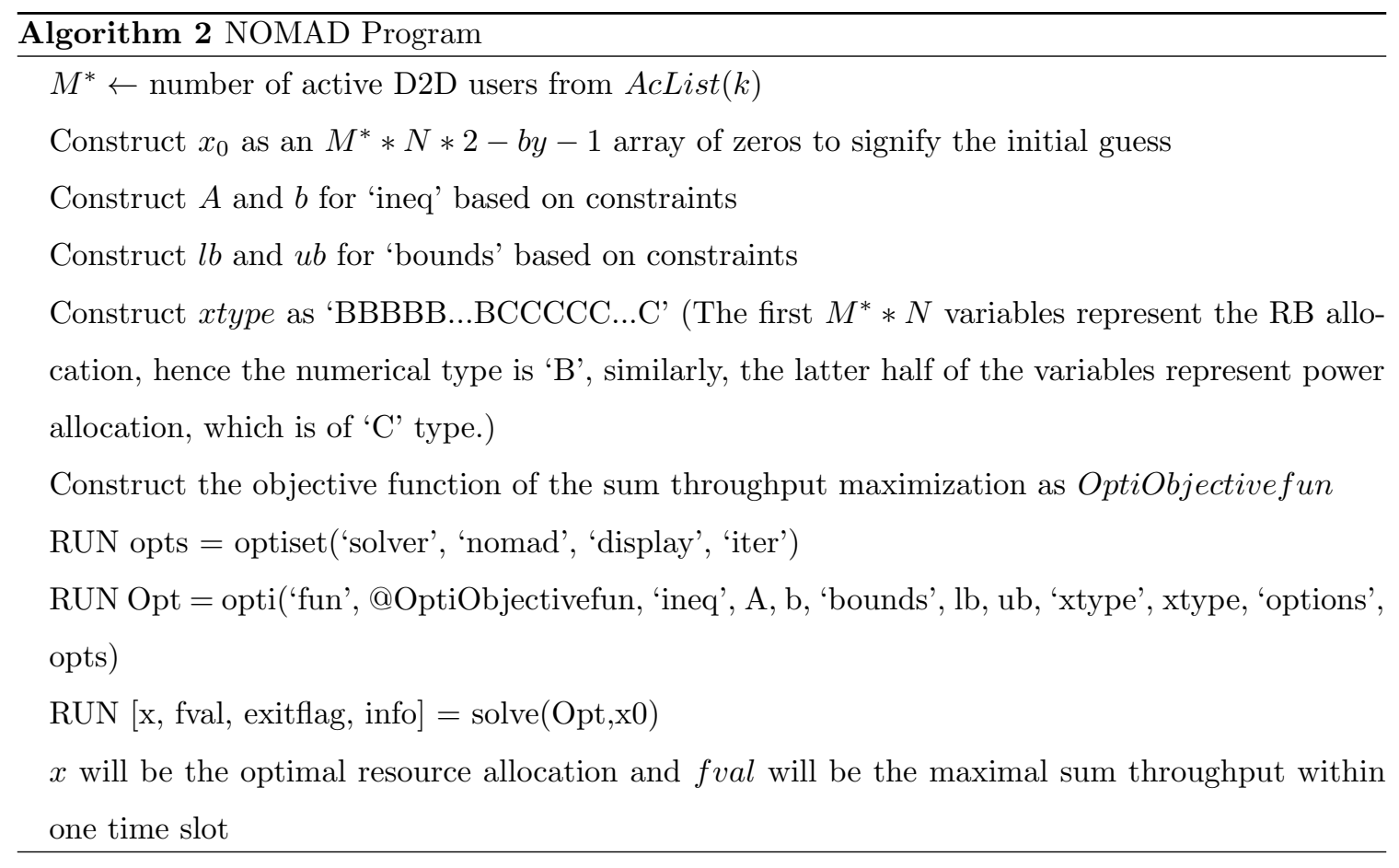

\subsection{Simulation Results}

Table 4.2: Simulation parameters.

\begin{tabular}{|cc|cc|}
\hline Symbol & Values & Symbol & Values \\
\hline$M$ & 4 & $K$ & 3600 \\
$N$ & $4-12$ & $E_{\max }$ & $5000 \mathrm{~J}$ \\
$\tau$ & $20 \mathrm{sec}$ & $E_{\text {up }}$ & $50 \mathrm{~J}$ \\
$\eta$ & $0.2-0.4$ & $E_{\text {low }}$ & $20 \mathrm{~J}$ \\
$R_{C} / W$ & $12-20 \mathrm{bit} / \mathrm{s} / \mathrm{Hz}$ & $P_{\max }$ & $10 \mathrm{dBm}$ \\
$N_{0}$ & $-170 \mathrm{dBm} / \mathrm{Hz}$ & $P_{H}$ & $46 \mathrm{dBm}$ \\
\hline
\end{tabular}

In this section, we provide numerical results of our simulations to show the performance of NOMAD algorithm. The network used in our simulation covers a cell area of radius $r=200 \mathrm{~m}$. The average distance between any D2D pair is $20 \mathrm{~m}$. System bandwidth $W=1.5 k H z$. Path loss constant 
$\kappa=10^{-2}$, path loss exponent $\alpha=3,4$ for cellular users and D2D pairs respectively. Because of the inner limitations of the solver, we are only able to run simulation up to 4 D2D users, with varying amounts of CUs and QoS requirements. The initial energy level for all batteries $E_{i, 0}=E_{\max }$. Other simulation parameters can be found in Table 4.2 .

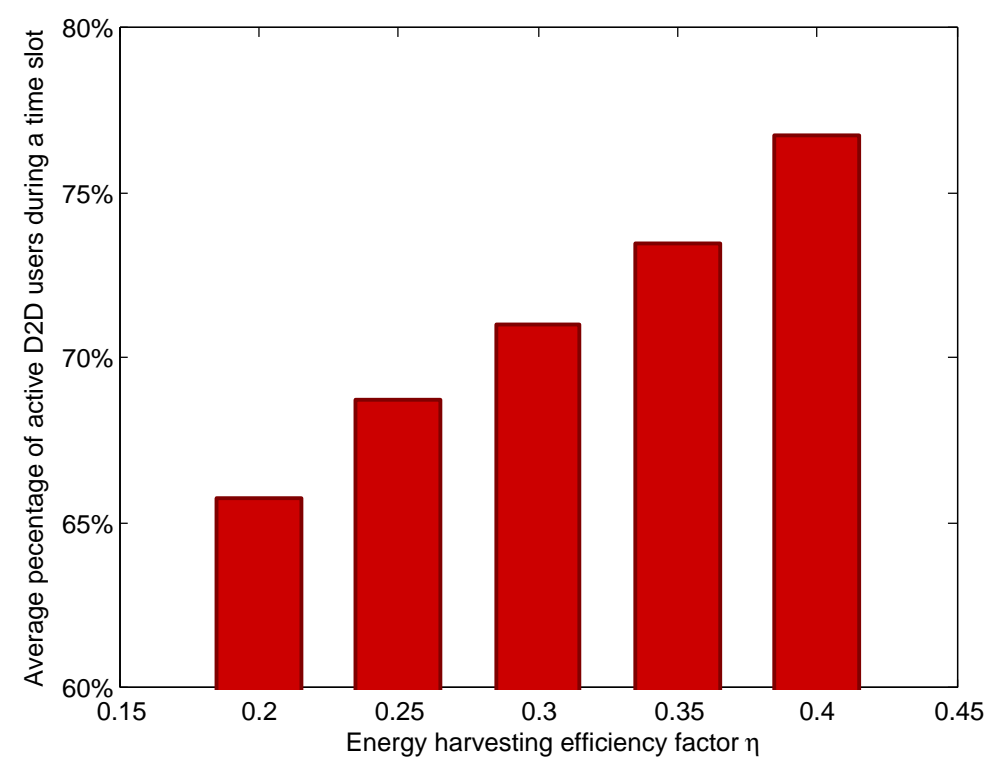

Figure 4.4: Relationship between EH efficiency factor and percentage of active D2D pairs.

Fig. 4.4 shows the relationship between EH efficiency factor $\eta$ and the average percentage of active D2D pairs in a time slot. From this graph, we can see clearly that NOMAD algorithm can lead to an average $65 \%-77 \%$ of active D2D users during a time slot. We can also intuitively observe how a higher $\eta$ will lead to more active D2D users due to the fact that faster charging rate will reduce the average time slots one D2D user spend on energy harvesting. It also verifies our primary estimation that higher $\eta$ will result in a higher percentage of active D2D pairs. In the following simulations of this chapter, we select the value of $\eta$ as 0.4 to generate more decent results. 


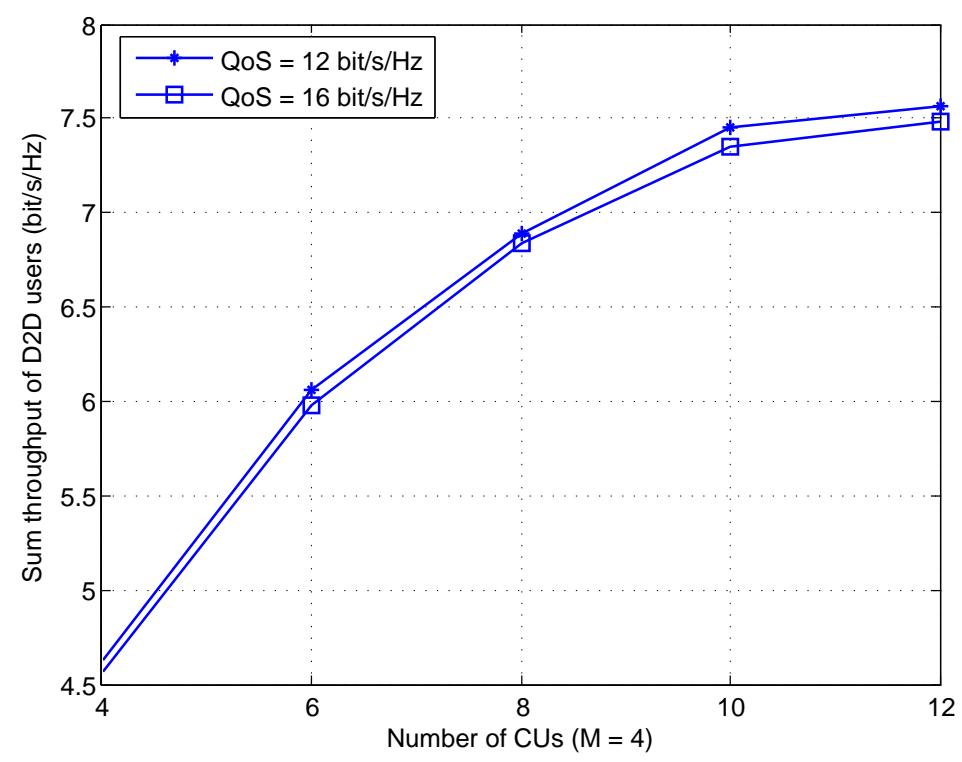

Figure 4.5: Sum throughput of D2D users on varying number of CUs.

Fig. 4.5 demonstrates the relationship between sum throughput of D2D users and number of CUs under different QoS requirements for CUs using NOMAD algorithm. As we can see that the effect of QoS on D2D sum throughput in this case (from $12 \mathrm{bit} / \mathrm{s} / \mathrm{Hz}$ to $16 \mathrm{bit} / \mathrm{s} / \mathrm{Hz}$ ) is not very salient, although a higher QoS for cellular users can lead to a slight decline in D2D sum throughput. We also discover that the sum throughput of D2D users will increase almost in proportion with the number of CUs from $4.5 \mathrm{bit} / \mathrm{s} / \mathrm{Hz}$ up until a threshold $(7.5 \mathrm{bit} / \mathrm{s} / \mathrm{hz})$ where it will remain relatively flat regardless of increasing CUs. The reason behind this can be attributed to two aspects. On one hand, more CUs can mean more RBs for spectrum use, which can lead to an increase in sum throughput given proper resource allocation techniques. On the other hand, since the transmit power of D2D users is limited to an upper threshold, the positive effect engendered by the extra RBs will come to a stop eventually. 


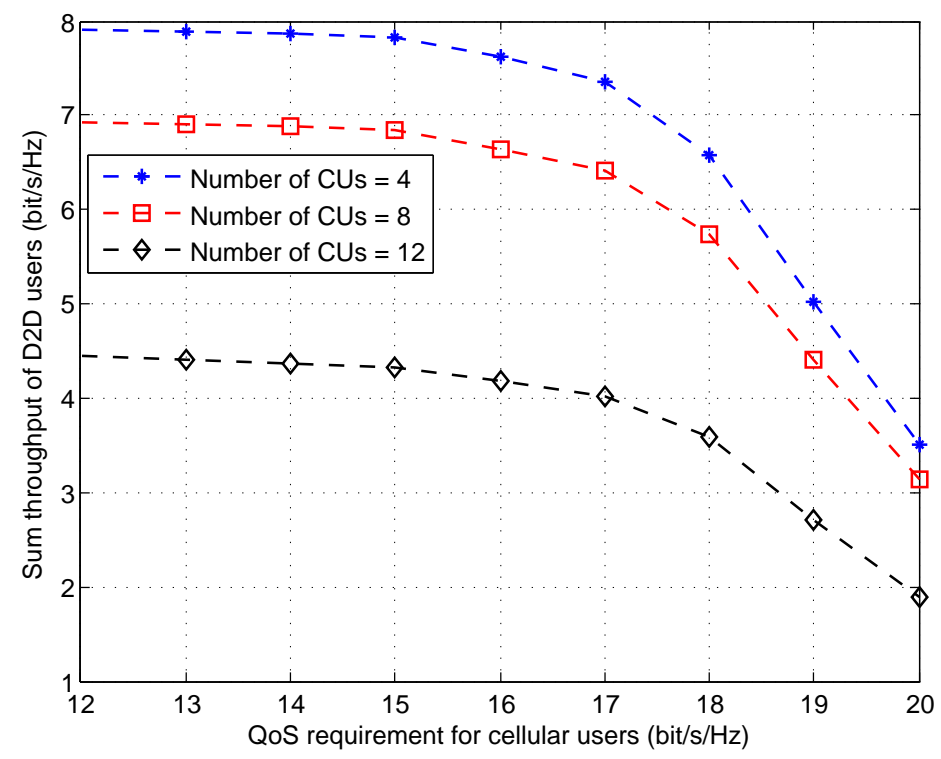

Figure 4.6: Sum throughput of D2D users on varying QoS requirements for CUs.

In Fig. 4.6, we showcase the connection between the sum throughput of D2D users and the QoS requirements for CUs under NOMAD algorithm. As we can see from the graph, when we increase the QoS requirements for CUs from $12 \mathrm{bit} / \mathrm{s} / \mathrm{Hz}$ to $16 \mathrm{bit} / \mathrm{s} / \mathrm{Hz}$, the sum throughout for D2D users is not affected very much; however, after $16 \mathrm{bit} / \mathrm{s} / \mathrm{Hz}$, any increase in QoS requirements will lead to obvious performance decay for D2D users. The reason we believe is due to the limitation of resource allocation. Proper resource allocation techniques can provide a maximal sum throughput for D2D users, thus under certain QoS requirements, the interference imposed on D2D users by CU can be neglected in terms of system performance. However, when the interference exceeds certain threshold, as generated by high QoS requirements, no resource allocation techniques can guarantee a perfect performance for D2D users. The critical value of QoS for CUs in this case is approximately $16 \mathrm{bit} / \mathrm{s} / \mathrm{Hz}$. 


\subsection{Summary}

In this chapter, we presented an energy harvesting assisted underlay D2D system model with centralized decision making approach. We then formulated an optimization problem, which is aimed at obtaining maximal sum throughput for D2D users. Because the arbitrary nature of the running time, we decided to focus on the the optimization within one time slot individually and then make decision about whether to transmit or harvest based on the remaining battery values at the beginning of the next time slot. The problem proved to be a MINLP one, which would be mathematically infeasible if we were to adopt exhaustive search. We employed the NOMAD solver in OPTI toolbox to solve this problem, which is a very popular tool for MINLP problems. Simulation results show that we can achieve an average 65\%-77\% active rate for D2D users. We can also produce decent sum throughput for D2D users under varying conditions of QoS requirements for cellular users and CU numbers. 


\section{Chapter 5}

\section{A Heuristic RB and Power Allocation Algorithm}

In the last chapter, we offered a solution using OPTI toolbox in the MATLAB. It was computationally complicated, although still manageable. In this chapter, we provide an alternative approach a heuristic resource block and power allocation algorithm - to solve the resource allocation problem given in (4.15), based on channel-based RB allocation with equal power distribution.

\subsection{Heuristic Algorithm}

As discussed in the last chapter, our heuristic algorithm still focuses on providing an optimal solution to the joint RB and power allocation problem within every time slot. The sum of all the optimal solutions will be the ultimate optimal solution apparently. At the beginning of every time slot $\tau_{k}$, we will check the energy level in all D2D pairs and put the ones that are able for data transmission in a list called AcList $(k)$. Since we assume that there are more RBs than D2D pairs, our iterations will start with D2D pairs. For all D2D pairs in the active list, we allocate one RB at a time to every D2D pair based on the exhaustive search to make sure that the D2D pairs underlaying RBs have the optimal throughput. We mark all the RBs that have been allocated as "occupied". Then using the similar technique, we go over all the RBs again, to allocate the unoccupied RBs to the D2D pairs. 


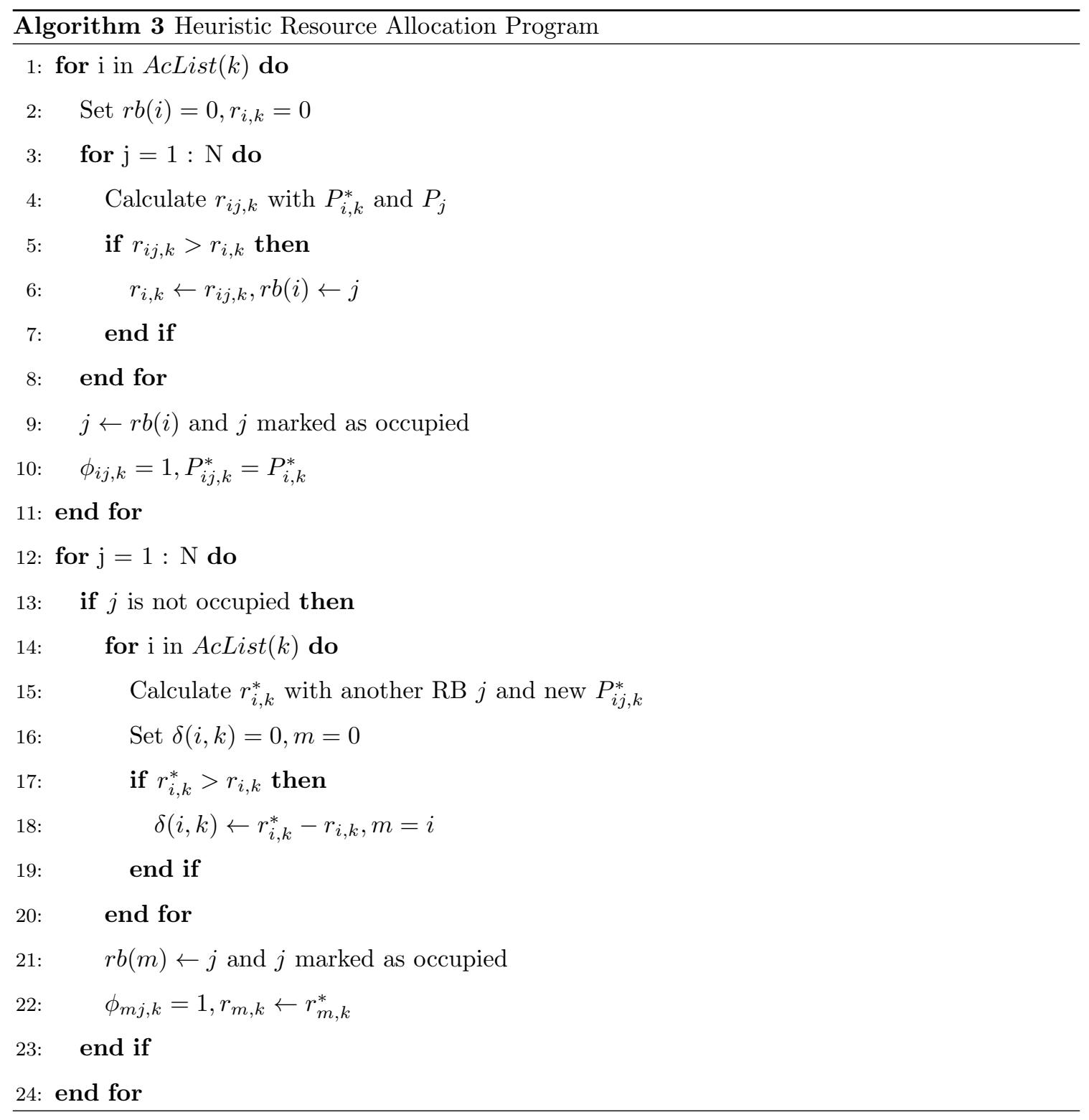

One aspect worth noticing is that a D2D pair can only take up another RB when doing so will lead to higher throughput. This is not always the case, since more RBs for one D2D pair means less transmit power over every RB as we we adopt an equal power allocation approach with fixed total power. We use $\delta(i, k)$ to signify the difference between before and after adding the RB to a D2D pair, and we will allocate the RB to the $\mathrm{D} 2 \mathrm{D}$ pair that will lead to the biggest positive impact on 
sum throughput. We will continue our approach until all RBs are shared to D2D pairs and marked as occupied. Then we will return the sum throughput for D2D users based on the optimal $P_{i j, k}$ and $\phi_{i j, k}$. Energy level will be automatically updated as well. The whole process is shown in Algorithm 1 and 3 for the purpose of clarity, except we would be calling Algorithm 3 instead of Algorithm 2 during the resource allocation process.

\subsection{Simulation Results}

In this section, we provide numerical results of our simulations and offer an evaluation of the heuristic resource allocation approach to show that it can achieve higher sum throughput while maintaining QoS performance for cellular users. Simulation parameters are almost the same with the ones carried out in the last chapter with NOMAD algorithm, except that in this case, we have more D2D users and cellular users due to the feasibility of the solution. Number of D2D users $M$ ranges from 6 to 15 and number of CUs $N$ ranges from 15 to 30.

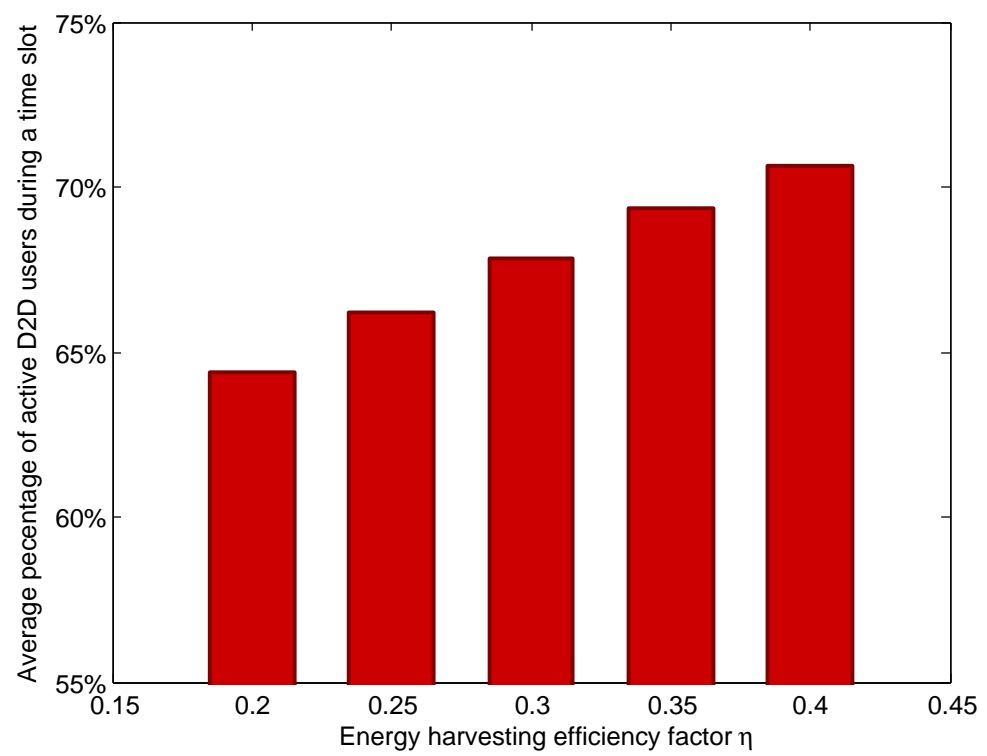

Figure 5.1: Relationship between EH efficiency factor and percentage of active D2D pairs.

Fig. 5.1 shows the relationship between EH efficiency factor $\eta$ and the average percentage of 
active D2D pairs in a time slot. As we can see in the graph, our heuristic approach will guarantee an average $60 \%-70 \%$ of active D2D users during a time slot. Similar to the results in last chapter, it also follows the pattern that higher $\eta$ will lead to higher active percentage of D2D pairs. We choose 0.4 for $\eta$ in our following simulations since it will produce optimal system performance.

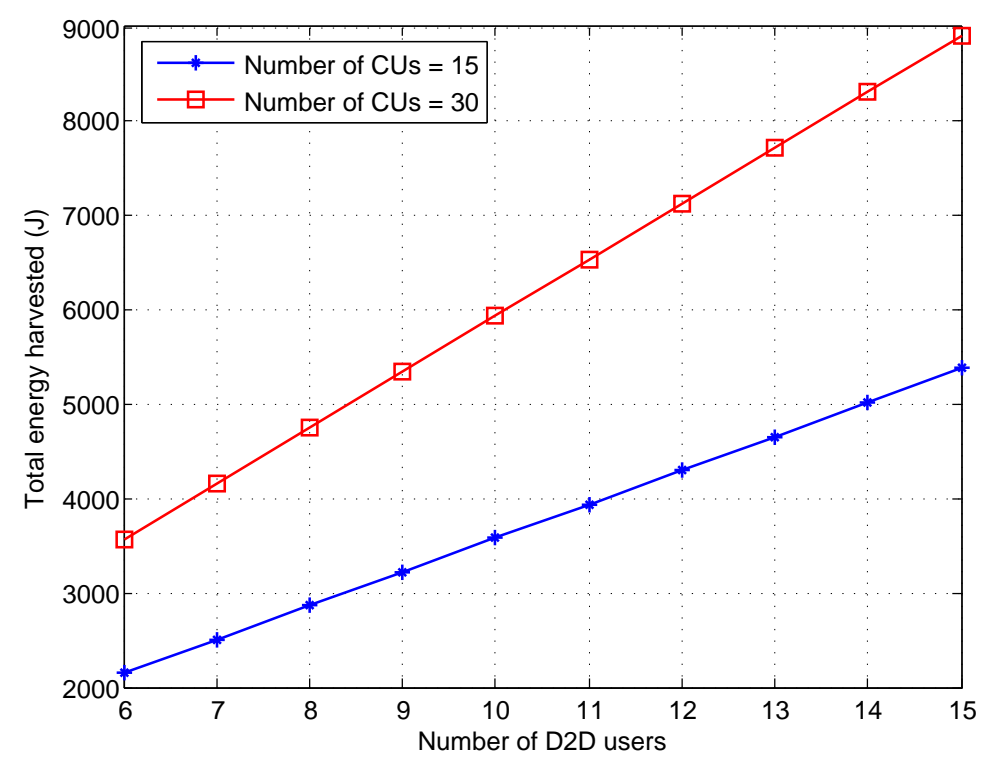

Figure 5.2: Total energy harvested on varying number of D2D users.

Fig. 5.2 demonstrates the relationship between total harvested energy and number of D2D users. As we can see, the growth is quasi-linear, this is because all D2D links harvest energy from the same source and the average distance will be the same eventually. In addition, we can notice that when there are 30 CUs in the system, D2D links can harvest more energy in general compared with the scenario of 15 CUs. The reason behind this is that when there are more CUs, each D2D link will on average have more RBs and higher transmit power. Therefore, their energy would be drained down below the lower threshold more quickly, thus leading to more energy charging slots. The more time slots a D2D link spends harvesting energy, the higher energy it ends up harvesting after a certain amount of time. 


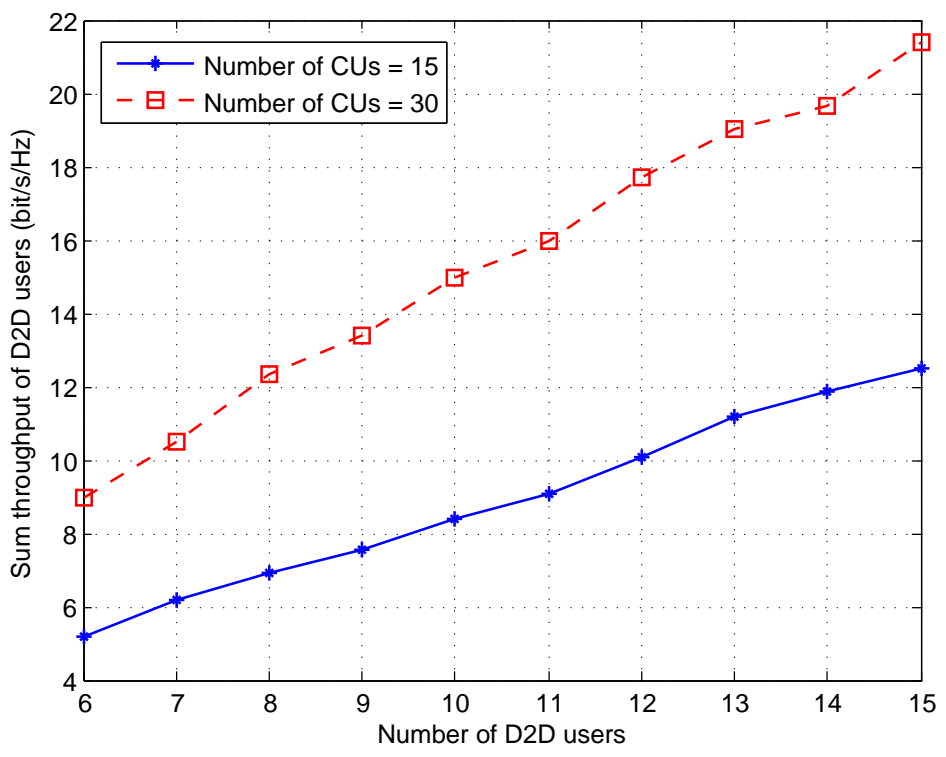

Figure 5.3: Sum throughput of D2D users on varying number of D2D users.

Fig. 5.3 illustrates the relationship between sum throughput of D2D users and number of D2D users under circumstances of varying number of CUs using two versions of the algorithm. We can see that when number of D2D users is relatively low, the increasing rate is similar according to the slope. However, when number of D2D users is high, it is clearly shown in the graph that the $N=30$ scenario outperforms the $N=15$ scenario even more. This is because more CUs than D2D pairs will eventually lead to more than one RB for every D2D pair, thus a much higher throughput. 


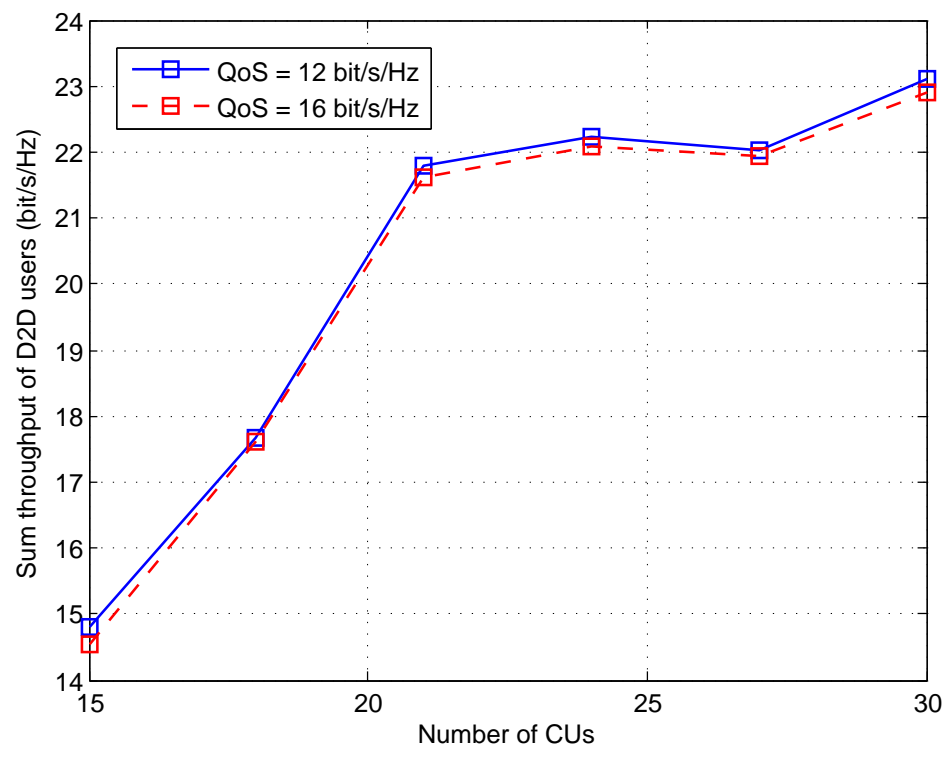

Figure 5.4: Sum throughput of D2D users on varying number of CUs.

Fig. 5.4 demonstrates the relationship between sum throughput of D2D users and number of CUs under different QoS requirements for CUs. We can discover that the sum throughput increases as the number of CUs increases. The reason behind is that more CUs create more available RBs to share with D2D users since each $\mathrm{CU}$ has its own independent RB that is orthogonal to the others. When the number of CUs is static, based on our observation, we discover that higher QoS requirement for CUs will lead to lower sum throughput for D2D users. This is predominantly due to the restricted transmit power of $\mathrm{D} 2 \mathrm{D}$ users since transmit power over a certain threshold will cause too much interference and compromise the set QoS requirement. 


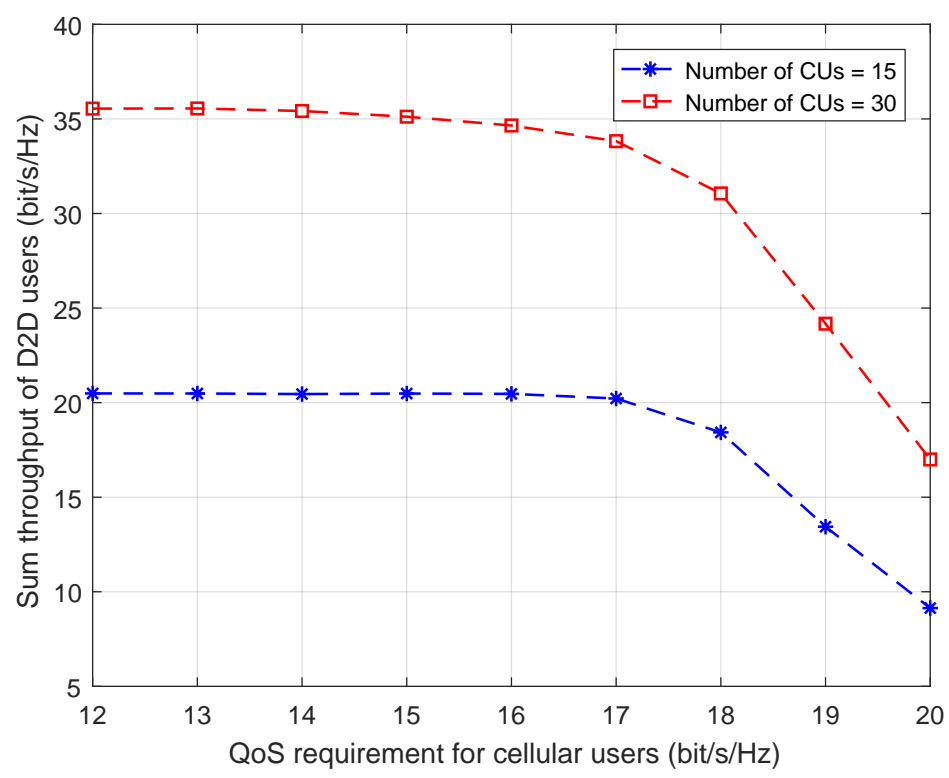

Figure 5.5: Sum throughput of D2D users on varying QoS requirements for CUs.

In Fig. 5.5, we explore the connection between the QoS requirements for CUs and the sum throughput of D2D users. The graphs capture the trade-off between CUs and D2D users. Obviously, we can observe that the sum throughput decreases as $R c / W$ increases from $12 \mathrm{bit} / \mathrm{s} / \mathrm{Hz}$ to 20 $\mathrm{bit} / \mathrm{s} / \mathrm{Hz}$. We also discover that starting from certain point (in this case, $R c / W=17 \mathrm{bit} / \mathrm{s} / \mathrm{Hz}$ ), the sum throughput will suffer intense affection. This is because higher QoS requirements for CUs call for lower transmit power allocated for D2D users to control the level of interference D2D transmission may impose on cellular devices. When transmit power of D2D users is low, attenuation and fading deteriorates and noise impacts become more obvious, leading the SINR of D2D users to plunge. Consequently, the sum throughput takes a dive. Another observation worth mentioning is that the more CUs we have in the system, the greater sum throughput the system can produce. The reason behind this is straightforward, since more CUs will provide more reusable orthogonal RBs for D2D users underlying cellular networks. 


\subsection{Results Comparison with NOMAD Algorithm}

In the last section, we presented a couple of results using our heuristic resource allocation algorithm. The algorithm is channel-based RB allocation with equal power allocation. In this section, we compare some of the results we generated from last chapter using NOMAD with the ones we obtained from last section using our own heuristic algorithm. The comparison will be focused on three aspects: percentage of active D2D users, sum throughput and time cost. We also include results of random $\mathrm{RB}$ allocation with equal power allocation as base values so that the pros and cons of both algorithms can be accentuated.

\subsubsection{Random RB Allocation with Equal Power Distribution}

In the random $\mathrm{RB}$ allocation approach, we allocate all RBs to all $\mathrm{D} 2 \mathrm{D}$ users in a purely random fashion as opposed to using maximal sum throughput as the criteria our heuristic channel-based $\mathrm{RB}$ allocation approach. Each RB has an equal probability of being allocated to any of the D2D users. Like in the heuristic algorithm, every RB will be allocated to one D2D pair through iteration and be marked as "occupied". If one D2D pair is allocated more than one RB, the transmit power will be shared equally over each RB. Note that we do not guarantee that each D2D pair will be allocated one RB since doing so will violate the principle of randomness. Our primary estimation is that both algorithms will outperform this random approach since no optimization techniques are included in this random resource allocation process. 


\subsubsection{Percentage of Active D2D Users}

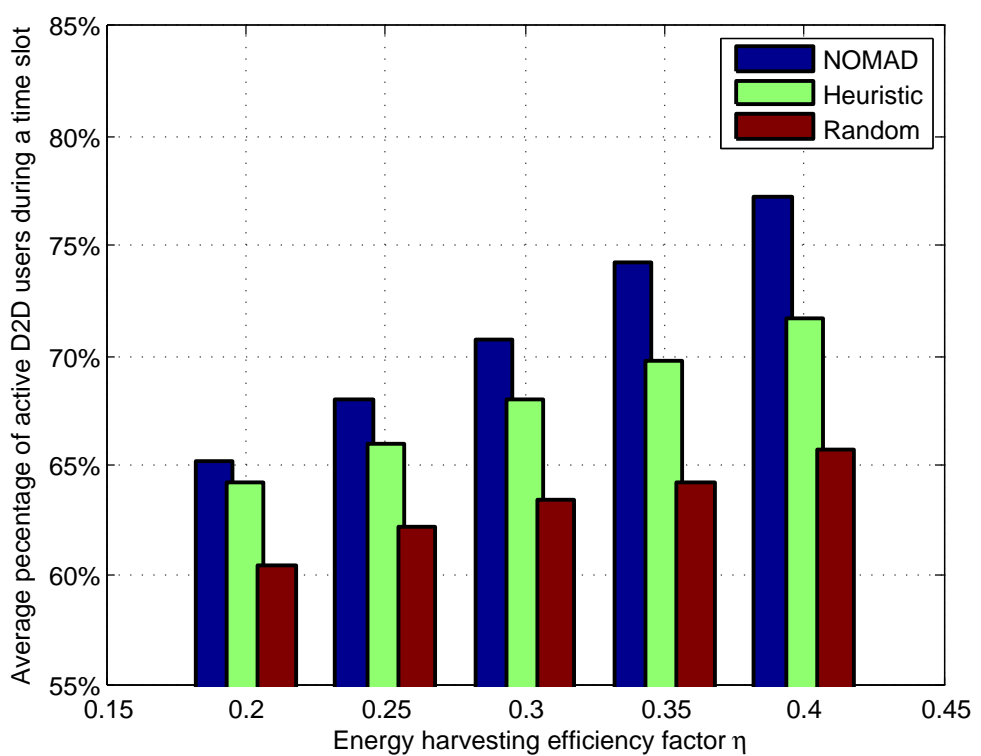

Figure 5.6: A comparison of average percentage of active D2D users between two algorithms

Fig. 5.6 compares the average percentage of active D2D users under two algorithms with random resource allocation. As we can see, both NOMAD and heuristic algorithm outperform random resource allocation. However, the advantage of NOMAD is more prominent as it outperforms the heuristic algorithm by $2 \%-7 \%$. The advantage seems to be more conspicuous as the value of $\eta$ increases. This makes sense in that our heuristic approach is aimed at providing an sub-optimal solution. Our power allocation approach cannot guarantee a higher energy efficiency than that in NOMAD algorithm, thus leading to some power resources under-utilized. And as $\eta$ increases, more energy will be harvested, which will lead to a widening gap between performances. However, we can also see that when $\eta$ is relatively low, the advantage may not be that obvious as in both cases energy efficiency will suffer from the system restrictions. In this, our approach offers a decent alternative at lower time cost. 


\subsubsection{Sum Throughput}

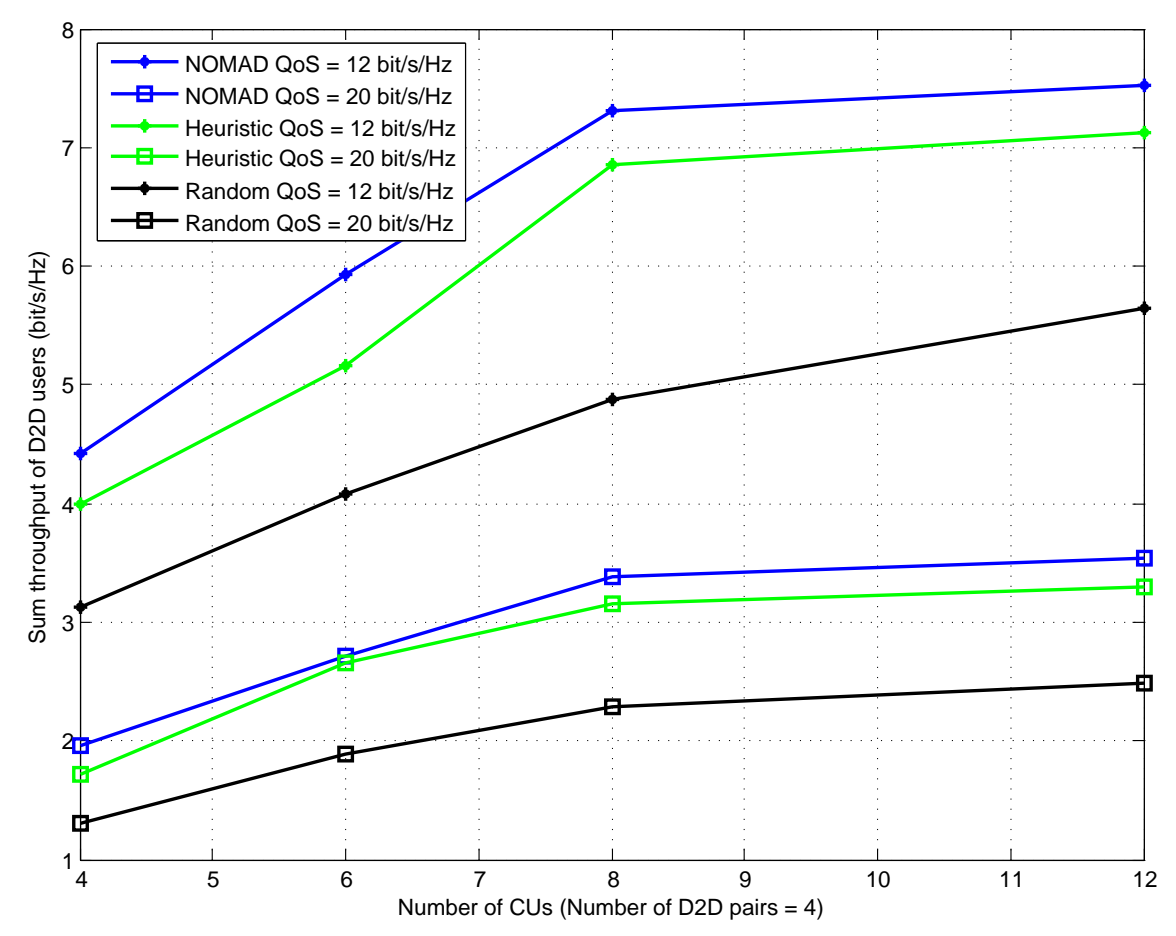

Figure 5.7: A comparison of D2D sum throughput under different algorithms

Fig. 5.7 enumerates the values of D2D sum throughput under different algorithms, different numbers of CUs and different QoS requirements with a fixed number of D2D users $(M=4)$. As we can see, both algorithms can lead to a higher sum throughput than random resource allocation, however; NOMAD algorithm can yield a slightly higher sum throughput in all cases when compared with our heuristic algorithm. The much worse performance of random approach proves that proper resource allocation, no matter how limited in its nature, can be beneficial to the system output. The advantage is bigger under the condition of lower QoS requirements because even though the increasing percentages are almost the same (around 10\%), the actual throughput gains are accruing. 
This verifies our analysis that NOMAD produces optimal solutions where as the heuristic approach produces sub-optimal solutions.

\subsubsection{Time Cost}

Even though NOMAD can produce better results than our heuristic algorithm, it is more time consuming. In our simulation, in order to solve the same problem, NOMAD will take dozens of hours while our own heuristic algorithm requires only minutes. Also our heuristic algorithm can deal with much more D2D users without worrying about scaling up the complexity of the problem, which is also true for the random approach. Since base station is the decision maker in our model, NOMAD algorithm might require much higher computational ability at BS's end and a significant system delay. However, when we have a small number of both D2D users and CUs (preferably equal amount), NOMAD can still have an edge over our heuristic algorithm. Fig. 5.8 recaps the details of time costs under different algorithms.

\begin{tabular}{|c|c|c|c|}
\hline Algorithms & NOMAD & Heuristic & Random \\
\hline $\begin{array}{c}\text { Maximal D2D } \\
\text { users }\end{array}$ & 4 & 15 & 15 \\
\hline $\begin{array}{c}\text { Time } \\
\text { Complexity }\end{array}$ & $\mathrm{O}(\mathrm{M}+\mathrm{M} \times \mathrm{N})^{2}$ & Linear & Linear \\
\hline Convergence & Not Always & Always & Always \\
\hline
\end{tabular}

Figure 5.8: A comparison of time cost under different algorithms

\subsection{Summary}

In this section, we provided an alternative solution to solve the optimization problem. NOMAD is effective for MINLP problems, however, there are limitations are the number of variables you can set up in your simulation. Because of this, we were only to run a simulation wth 4 D2D users in the last chapter. In this chapter, we produced more results with our heuristic joint power and RB algorithm. 
We chose equal power allocation and channel-based approach to select optimal RB for a particular D2D user. We also adopted a random RB approach with equal power distribution as a baseline for the results comparison of two solutions. We can see that both the optimal solution and the suboptimal solution can outperform random approach significant in almost all simulations. However, NOMAD as the optimal solution has advantage in many cases. As we expected, the NOMAD algorithm proved to be slightly better than our heuristic method in terms of both percentage of average active D2D users and sum throughput, though at a much higher time cost. 


\section{Chapter 6}

\section{Conclusions and Future work}

This thesis mainly focused on the joint resource allocation problem in an energy harvesting aided underlay D2D system. Since the objective function is a mixed integer nonlinear programming problem (MINLP), two solutions are proposed. The first method adopts the NOMAD algorithm embedded in OPTI tool box in MATALB while the second one is a heuristic algorithm based on the combination of distance-based resource block allocation and equal power distribution. In this chapter, we recapitulate the main features and effects of these two methods.

\subsection{Conclusion}

NOMAD and our heuristic algorithm both prove to be capable of solving our optimization problem, and each of them has their own advantages and disadvantages. The advantages of NOMAD algorithm include:

- higher sum throughput under the same condition compared with our heuristic algorithm;

- higher percentage of active D2D users in a time slot under same energy harvesting efficiency eta;

- easy to expand to include more constraints.

The disadvantages of NOMAD algorithm include: 
- usually takes more time to produce a result compared with our heuristic algorithm;

- the feasible solution is not guaranteed as the simulation might not converge;

- has limitations on the number of D2D users we can use in simulation.

Similarly, we can deduce that our own heuristic can produce sub-optimal solution in less computational time and be applicable to cases of more D2D users.

\subsection{Future Work}

In this thesis, we explored energy harvesting in D2D communications. Our energy harvesting transmitter is located at the BS, so the BS acts as the centralized decision maker to initiate or pause energy harvesting for D2D users at the beginning of each time slot based on the remaining energy level. For future work, it would be interesting to discuss the deployment of multiple wireless energy harvesting transmitters in the coverage cell and how it will affect D2D communications. We've already seen similar work in WSN, so this topic might be a good place to start. Another aspect worth exploring is energy harvesting assisted D2D in heterogeneous networks (HetNets). Few similar work have been published in this field. 


\section{Bibliography}

[1] X. Wu, S. Tavildar, S. Shakkottai, T. Richardson, J. Li, R. Laroia, and A. Jovicic, "Flashlinq: A synchronous distributed scheduler for peer-to-peer ad hoc networks," IEEE/ACM Transactions on Networking, vol. 21, pp. 1215-1228, Aug 2013.

[2] P. Cheng, L. Deng, H. Yu, Y. Xu, and H. Wang, "Resource allocation for cognitive networks with d2d communication: An evolutionary approach," in Wireless Communications and Networking Conference (WCNC), 2012 IEEE, pp. 2671-2676, IEEE, 2012.

[3] Y.-L. Chung and M.-Y. Lin, "A power-saving resource allocation algorithm for d2d-assisted cellular networks," in Innovative Computing Technology (INTECH), 2014 Fourth International Conference on, pp. 176-179, IEEE, 2014.

[4] H. Zhou, Y. Ji, J. Li, and B. Zhao, "Joint mode selection, mcs assignment, resource allocation and power control for $\mathrm{d} 2 \mathrm{~d}$ communication underlaying cellular networks," in Wireless Communications and Networking Conference (WCNC), 2014 IEEE, pp. 1667-1672, IEEE, 2014.

[5] L. AlWreikat, R. Chai, and O. M. Abu-Sharkh, "Energy-efficiency based resource allocation for d2d communication and cellular networks," in Big Data and Cloud Computing (BdCloud), 2014 IEEE Fourth International Conference on, pp. 722-728, IEEE, 2014.

[6] Z. Zhou, M. Dong, K. Ota, G. Wang, and L. Yang, "Energy-efficient resource allocation for d2d communications underlaying cloud-ran based lte-a networks,"

[7] K. Yang, S. Martin, L. Boukhatem, J. Wu, and X. Bu, "Energy-efficient resource allocation for device-to-device communications overlaying lte networks," 
[8] T. D. Hoang, L. B. Le, and T. Le-Ngoc, "Energy-efficient resource allocation for d2d communications in cellular networks," in Communications (ICC), 2015 IEEE International Conference on, pp. 2251-2256, IEEE, 2015.

[9] D. Tsolkas, E. Liotou, N. Passas, and L. Merakos, "A graph-coloring secondary resource allocation for $\mathrm{d} 2 \mathrm{~d}$ communications in lte networks," in Computer Aided Modeling and Design of Communication Links and Networks (CAMAD), 2012 IEEE 17th International Workshop on, pp. 56-60, IEEE, 2012.

[10] E. Liotou, D. Tsolkas, N. Passas, and L. Merakos, "Ant colony optimization for resource sharing among d2d communications," in Computer Aided Modeling and Design of Communication Links and Networks (CAMAD), 2014 IEEE 19th International Workshop on, pp. 360-364, IEEE, 2014.

[11] J.-W. Kao, Y.-Y. Shih, A.-C. Pang, and Y.-C. Lin, "Radio resource allocation for d2d-assisted full-duplex cellular networks," in Ubiquitous and Future Networks (ICUFN), 2015 Seventh International Conference on, pp. 721-726, IEEE, 2015.

[12] B. Peng, C. Hu, T. Peng, and W. Wang, "Optimal resource allocation for multi-d2d links underlying ofdma-based communications," in Wireless Communications, Networking and Mobile Computing (WiCOM), 2012 8th International Conference on, pp. 1-4, IEEE, 2012.

[13] M. Hasan and E. Hossain, "Distributed resource allocation for relay-aided device-to-device communication: A message passing approach," Wireless Communications, IEEE Transactions on, vol. 13, no. 11, pp. 6326-6341, 2014.

[14] J. Huang, Y. Zhao, and K. Sohraby, "Game-theoretic resource allocation for intercell deviceto-device communication underlaying cellular network," in Wireless Research Collaboration Symposium (NWRCS), 2014 National, pp. 79-83, IEEE, 2014.

[15] W. Sun, D. Yuan, E. G. Strom, and F. Brannstrom, "Resource sharing and power allocation for d2d-based safety-critical v2x communications," in Communication Workshop (ICCW), 2015 IEEE International Conference on, pp. 2399-2405, IEEE, 2015. 
[16] A. Bhardwaj and S. Agnihotri, "A resource allocation scheme for device-to-device multicast in cellular networks," in Personal, Indoor, and Mobile Radio Communications (PIMRC), 2015 IEEE 26th Annual International Symposium on, pp. 1498-1502, IEEE, 2015.

[17] R. Yin, G. Yu, C. Zhong, and Z. Zhang, "Distributed resource allocation for d2d communication underlaying cellular networks," in Communications Workshops (ICC), 2013 IEEE International Conference on, pp. 138-143, IEEE, 2013.

[18] J. Zhang, G. Wu, W. Xiong, Z. Chen, and S. Li, "Utility-maximization resource allocation for device-to-device communication underlaying cellular networks," in Globecom Workshops (GC Wkshps), 2013 IEEE, pp. 623-628, IEEE, 2013.

[19] T. D. Hoang, L. B. Le, and T. Le-Ngoc, "Joint subchannel and power allocation for d2d communications in cellular networks," in Wireless Communications and Networking Conference (WCNC), 2014 IEEE, pp. 1338-1343, IEEE, 2014.

[20] W. Wu and Y. Zhang, "Dedicated resource allocation for $\mathrm{d} 2 \mathrm{~d}$ communications in cellular systems employing ffr," in Wireless Communications and Signal Processing (WCSP), 2014 Sixth International Conference on, pp. 1-6, IEEE, 2014.

[21] H. Nguyen, M. Hasegawa, and W.-J. Hwang, "Distributed resource allocation for d2d communications underlay cellular networks,"

[22] J. Huang, Y. Zhao, and K. Sohraby, "Resource allocation for intercell device-to-device communication underlaying cellular network: A game-theoretic approach," in Computer Communication and Networks (ICCCN), 2014 23rd International Conference on, pp. 1-8, IEEE, 2014.

[23] N. Chen, H. Tian, and Z. Wang, "Resource allocation for intra-cluster d2d communications based on kuhn-munkres algorithm," in Vehicular Technology Conference (VTC Fall), 2014 IEEE 80th, pp. 1-5, IEEE, 2014.

[24] Y. Zhao, B. Pelletier, and D. Pani, "Resource allocation schemes for d2d communications," in Systems, Applications and Technology Conference (LISAT), 2014 IEEE Long Island, pp. 1-5, IEEE, 2014. 
[25] W. Xing, N. Wang, C. Wang, F. Liu, and Y. Ji, "Resource allocation schemes for d2d communication used in vanets," in Vehicular Technology Conference (VTC Fall), 2014 IEEE 80th, pp. 1-6, IEEE, 2014.

[26] V. Venkatasubramanian, F. Sanchez Moya, and K. Pawlak, "Centralized and decentralized multi-cell $\mathrm{d} 2 \mathrm{~d}$ resource allocation using flexible $\mathrm{ul} / \mathrm{dl}$ tdd," in Wireless Communications and Networking Conference Workshops (WCNCW), 2015 IEEE, pp. 305-310, IEEE, 2015.

[27] J. Zheng, R. Chen, and Y. Zhang, "Service time prediction based dynamic resource allocation for device-to-device communication underlaying cellular networks," in Global Communications Conference (GLOBECOM), 2014 IEEE, pp. 4928-4934, IEEE, 2014.

[28] H. Zhu, Y. Cao, W. Wang, B. Liu, and T. Jiang, "Qoe-aware resource allocation for adaptive device-to-device video streaming," Network, IEEE, vol. 29, no. 6, pp. 6-12, 2015.

[29] L. Su, Y. Ji, P. Wang, and F. Liu, "Resource allocation using particle swarm optimization for d2d communication underlay of cellular networks," in Wireless Communications and Networking Conference (WCNC), 2013 IEEE, pp. 129-133, IEEE, 2013.

[30] R. Zhang, X. Cheng, L. Yang, and B. Jiao, "Interference graph-based resource allocation (ingra) for d2d communications underlaying cellular networks," Vehicular Technology, IEEE Transactions on, vol. 64, no. 8, pp. 3844-3850, 2015.

[31] X. Wu, Y. Chen, X. Yuan, and M. E. Mkiramweni, "Joint resource allocation and power control for cellular and device-to-device multicast based on cognitive radio," Communications, IET, vol. 8, no. 16, pp. 2805-2813, 2014.

[32] F. Malandrino, Z. Limani, C. Casetti, and C.-F. Chiasserini, "Interference-aware downlink and uplink resource allocation in hetnets with d2d support," Wireless Communications, IEEE Transactions on, vol. 14, no. 5, pp. 2729-2741, 2015.

[33] C.-W. Yeh, G.-Y. Lin, M.-J. Shih, and H.-Y. Wei, "Centralized interference-aware resource allocation for device-to-device broadcast communications," in Internet of Things (iThings), 2014 IEEE International Conference on, and Green Computing and Communications (GreenCom), IEEE and Cyber, Physical and Social Computing (CPSCom), IEEE, pp. 304-307, IEEE, 2014. 
[34] S. Maghsudi and S. Stanczak, "Hybrid centralized-distributed resource allocation for deviceto-device communication underlaying cellular networks," 2015.

[35] S.-A. Ciou, J.-C. Kao, C. Y. Lee, and K.-Y. Chen, "Multi-sharing resource allocation for device-to-device communication underlaying 5g mobile networks," in Personal, Indoor, and Mobile Radio Communications (PIMRC), 2015 IEEE 26th Annual International Symposium on, pp. 1509-1514, IEEE, 2015.

[36] X. Cai, J. Zheng, Y. Zhang, and H. Murata, "A capacity oriented resource allocation algorithm for device-to-device communication in mobile cellular networks," in Communications (ICC), 2014 IEEE International Conference on, pp. 2233-2238, IEEE, 2014.

[37] S. Fowler, Y. Li, A. Pollastro, and S. Napoli, "Simple network design and power allocation for 5g device-to-device communication," in Computer Aided Modeling and Design of Communication Links and Networks (CAMAD), 2014 IEEE 19th International Workshop on, pp. 203-207, IEEE, 2014.

[38] A. Pratap and R. Misra, "Resource sharing in $\mathrm{d} 2 \mathrm{~d}$ communication underlaying cellular ltea networks," in Advances in Computing, Communications and Informatics (ICACCI), 2015 International Conference on, pp. 103-109, IEEE, 2015.

[39] S. Hamdoun, A. Rachedi, and Y. Ghamri-Doudane, "Radio resource sharing for mtc in lte-a: An approach based on the bipartite graph," in Protocol Engineering (ICPE) and International Conference on New Technologies of Distributed Systems (NTDS), 2015 International Conference on, pp. 1-6, IEEE, 2015.

[40] S. Cicalò and V. Tralli, "Joint admission control and resource allocation for d2d communications with qos constraints," in 2015 IEEE Globecom Workshops (GC Wkshps), pp. 1-6, IEEE, 2015.

[41] R. An, J. Sun, S. Zhao, and S. Shao, "Resource allocation scheme for device-to-device communication underlying lte downlink network," in Wireless Communications 83 Signal Processing (WCSP), 2012 International Conference on, pp. 1-5, IEEE, 2012. 
[42] T. D. Hoang, L. B. Le, and T. Le-Ngoc, "Resource allocation for d2d communications under proportional fairness," in Global Communications Conference (GLOBECOM), 2014 IEEE, pp. 1259-1264, IEEE, 2014.

[43] Y. Sun, T. Wang, L. Song, and Z. Han, "Efficient resource allocation for mobile social networks in $\mathrm{d} 2 \mathrm{~d}$ communication underlaying cellular networks," in Communications (ICC), 2014 IEEE International Conference on, pp. 2466-2471, IEEE, 2014.

[44] Q. Gao, R. Zhao, W. Chen, Y. Peng, B. Guo, and S. Sun, "Radio resource management of d2d communication," in Communication Systems (ICCS), 2014 IEEE International Conference on, pp. 6-10, IEEE, 2014.

[45] A. H. Sakr and E. Hossain, "Cognitive and energy harvesting-based d2d communication in cellular networks: Stochastic geometry modeling and analysis," IEEE Transactions on Communications, vol. 63, pp. 1867-1880, May 2015.

[46] H. H. Yang, J. Lee, and T. Q. S. Quek, "Heterogeneous cellular network with energy harvesting-based d2d communication," IEEE Transactions on Wireless Communications, vol. 15, pp. 1406-1419, Feb 2016.

[47] Z. Zhou, G. Ma, C. Xu, and Z. Chang, "A game-theoretical approach for green power allocation in energy-harvesting device-to-device communications," in 2016 IEEE 83rd Vehicular Technology Conference (VTC Spring), pp. 1-5, May 2016.

[48] J. Ding, L. Jiang, and C. He, "Dynamic spectrum allocation for energy harvesting-based underlaying d2d communication," in 2016 IEEE 83rd Vehicular Technology Conference (VTC Spring), pp. 1-5, May 2016.

[49] S. Gupta, R. Zhang, and L. Hanzo, "Energy harvesting aided device-to-device communication underlaying the cellular downlink," IEEE Access, vol. PP, no. 99, pp. 1-1, 2016.

[50] U. Saleem, H. K. Qureshi, S. Jangsher, and M. Saleem, "Transmission power management for throughput maximization in harvesting enabled d2d network," in 2016 IEEE Symposium on Computers and Communication (ISCC), pp. 1078-1083, June 2016. 
[51] L. Jiang, H. Tian, Z. Xing, K. Wang, K. Zhang, S. Maharjan, S. Gjessing, and Y. Zhang, "Social-aware energy harvesting device-to-device communications in 5g networks," IEEE Wireless Communications, vol. 23, pp. 20-27, August 2016.

[52] P. Lakhlan and A. Trivedi, "Energy harvesting-based two-hop d2d communication in cellular networks," in 2016 International Conference on Advances in Computing, Communications and Informatics (ICACCI), pp. 328-332, Sept 2016.

[53] M. L. Ku, W. Li, Y. Chen, and K. J. R. Liu, "Advances in energy harvesting communications: Past, present, and future challenges," IEEE Communications Surveys Tutorials, vol. 18, pp. 1384-1412, Secondquarter 2016.

[54] S. Reddy and C. R. Murthy, "Profile-based load scheduling in wireless energy harvesting sensors for data rate maximization," in 2010 IEEE International Conference on Communications, pp. 1-5, May 2010.

[55] W. Ejaz, M. Naeem, M. Basharat, A. Anpalagan, and S. Kandeepan, "Efficient wireless power transfer in software-defined wireless sensor networks," IEEE Sensors Journal, vol. 16, pp. 74097420 , Oct 2016. 